\title{
Forschungsbericht
}

Jörg Echternkamp, Dieter H. Kollmer, Thorsten Loch, Ralf Vollmuth und Rüdiger Wenzke

\section{Deutsche Militärgeschichte von 1945 bis 1990 im internationalen Kontext}

\author{
Bilanz und Perspektiven der Forschung
}

DOI 10.1515/mgzs-2017-0005

Die deutsche institutionalisierte Militärgeschichtsforschung kann in diesem Jahr auf eine ertragreiche sechzigjährige Geschichte zurückblicken. Seit seiner Gründung 1957 hat das Militärgeschichtliche Forschungsamt (MGFA), anfangs von Freiburg im Breisgau und später von Potsdam aus, die militärgeschichtliche Forschung in Deutschland entscheidend geprägt und mitbestimmt. ${ }^{1}$ Seit 2013 führt die Nachfolgeeinrichtung des MGFA, das Zentrum für Militärgeschichte und Sozialwissenschaften der Bundeswehr (ZMSBw), die umfangreiche Forschungsarbeit auf dem Gebiet der deutschen Militärgeschichte fort. Dazu gehört die Beschäftigung mit der Militärgeschichte der Bundesrepublik Deutschland und

\footnotetext{
150 Jahre Militärgeschichtliches Forschungsamt. Eine Chronik. Hrsg. vom Militärgeschichtlichen Forschungsamt (MGFA). Bearb. von Martin Rink, Berlin 2007; Jörg Echternkamp, Auftrag: Forschung. Die Bundeswehr, das Verteidigungsministerium und die Aufarbeitung der NS-Vergangenheit im Systemkonflikt. In: Zeitgeschichte-online, Juni 2015, <www.zeitgeschichte-online. de/thema/auftrag-forschung (letzter Zugriff 18.1.2017).

\begin{abstract}
Anmerkung: Die Autoren sind Angehörige des Forschungsbereichs »Militärgeschichte nach 1945« am Zentrum für Militärgeschichte und Sozialwissenschaften der Bundeswehr (ZMSBw), Potsdam, mit Ausnahme von Ralf Vollmuth, der Beauftragter des Inspekteurs des Sanitätsdienstes für Geschichte, Theorie und Ethik der Wehrmedizin am ZMSBw ist. Der Bilanzzeitraum des Aufsatzes konzentriert sich auf die Zeit von 2005 bis 2016. Die bibliografischen Angaben stellen eine Auswahl selbstständiger Publikationen dar.
\end{abstract}

Kontakt: Jörg Echternkamp, ZMSBw, Potsdam, E-Mail: JoergEchternkamp@bundeswehr.org Dieter H. Kollmer, ZMSBw, Potsdam, E-Mail: DieterKollmer@bundeswehr.org Thorsten Loch, ZMSBw, Potsdam, E-Mail: ThorstenLoch@bundeswehr.org Ralf Vollmuth, ZMSBw, Potsdam, E-Mail: Ralf1Vollmuth@bundeswehr.org Rüdiger Wenzke, ZMSBw, Potsdam, E-Mail: RuedigerWenzke@bundeswehr.org 
der DDR sowie mit der Geschichte der beiden Bündnisse, der NATO und dem Warschauer Pakt bis 1990.

\section{Zur Genese und Relevanz der militärgeschichtlichen Forschung}

Im Mittelpunkt der Forschung für die Zeit nach 1945 stand und steht die Militärgeschichte der Bundesrepublik Deutschland bis 1990. Sie umfasst im weitesten Sinne die außen-, sicherheits- und militärpolitischen sowie die militärischen Aspekte des westdeutschen Staates im weltumspannenden, antagonistischen Konflikt des Kalten Krieges. In einem engeren Sinne zielt sie jedoch auf die Geschichte der Streitkräfte der Bundesrepublik, mithin die Geschichte der Bundeswehr.

Bereits Anfang der 1970er Jahre begannen in Freiburg Forschungen zur Entstehungsgeschichte der westdeutschen Streitkräfte. Eine Projektgruppe, aus der später der eigenständige MGFA-Forschungsbereich »Geschichte der Bundeswehr « hervorging, durchforstete Akten, befragte Zeitzeugen und veröffentlichte erste wegweisende Publikationen. ${ }^{2}$ Heute gilt die Geschichte der Bundeswehr zumindest bis in die 1970er Jahre hinein als insgesamt gut erforscht. ${ }^{3}$

Dennoch ist sie in der allgemeinen Geschichtsschreibung der Bundesrepublik Deutschland ein Randgebiet geblieben. ${ }^{4} \mathrm{Zu}$ diesem Befund passt es, dass in der Militärgeschichtlichen Zeitschrift (MGZ) seit 2005 keine größere Debatte über die Verortung der Geschichte der Bundeswehr innerhalb der Geschichte der Bundes-

2 Vgl. u.a. Aspekte der deutschen Wiederbewaffnung bis 1955, Boppard a.Rh. 1975 (= Militärgeschichte seit 1945, 1); Roland G. Foerster, Christian Greiner, Georg Mayer, Hans-Jürgen Rautenberg und Norbert Wiggershaus, Von der Kapitulation bis zum Pleven-Plan, München 1982 (= Anfänge westdeutscher Sicherheitspolitik 1945 bis 1956 [AWS], 1).

3 Vgl. u.a. Die Bundeswehr 1955 bis 2005. Rückblende - Einsichten - Perspektiven. Im Auftrag des MGFA hrsg. von Frank Nägler, München 2007 (= Sicherheitspolitik und Streitkräfte der Bundesrepublik Deutschland, 7); Die Zeit nach 1945. Armeen im Wandel. Im Auftrag des MGFA hrsg. von Karl-Volker Neugebauer, München 2008 (= Grundkurs deutsche Militärgeschichte, 3); Sonderfall Bundeswehr? Streitkräfte in nationalen Perspektiven und im internationalen Vergleich. Im Auftrag des ZMSBw hrsg. von Heiner Möllers und Rudolf J. Schlaffer, München 2014 (= Sicherheitspolitik und Streitkräfte der Bundesrepublik Deutschland, 12); Martin Rink, Die Bundeswehr 1950/55-1989, Berlin, Boston 2015 (= Militärgeschichte kompakt, 6); Rudolf J. Schlaffer und Marina Sandig, Die Bundeswehr 1955 bis 2015: Sicherheitspolitik und Streitkräfte in der Demokratie, Freiburg i.Br., Berlin, Wien 2015.

4 Dominik Geppert, Die Bundeswehr in der Geschichtsschreibung der Bundesrepublik. In: Sonderfall Bundeswehr? (wie Anm. 3), S. 35. 
republik geführt wurde. Anders als in den Jahrgängen der 1970er und 1980er Jahre erschienen in ihren 20 Bänden seit 2005 nur wenige Beiträge, die sich mit der Entwicklung westdeutscher Sicherheitspolitik oder ihrer Streitkräfte befassen. Wirft man einen vergleichenden Blick in die Vierteljahrshefte für Zeitgeschichte oder in die militärgeschichtliche Buchreihe »Krieg in der Geschichte«, ergibt sich ein ähnliches Bild. ${ }^{5}$

Diese geringe Beachtung der Militärgeschichte der Bundesrepublik außerhalb des MGFA/ZMSBw mag dem nicht immer leichten Zugang zu Aktenmaterial des Bundesministeriums der Verteidigung im Bundesarchiv, Abteilung Militärarchiv, in Freiburg geschuldet sein. Viele der dort lagernden Akten sind unverändert als Verschlusssache (VS) »eingestuft« oder stehen wie v. a. Personalakten der Forschung aus Gründen des Persönlichkeits- und Datenschutzes nicht vollumfänglich zur Verfügung. Abhilfe schafft derzeit ein Beschluss der Bundesregierung vom September 2009. Das Bundeskabinett hatte die grundsätzliche Offenlegung aller noch eingestuften alten Verschlusssachen des Bundes und seiner Ministerien (Alt-VS) bis einschließlich 1994 nach vorheriger Prüfung durch die jeweiligen Ministerien beschlossen. Eine ad hoc eingerichtete Arbeitsgruppe des MGFA/ZMSBw sichtet seit 2011 entsprechende Akten des Verteidigungsministeriums mit dem Ziel ihrer Offenlegung. Dennoch wird auf absehbare Zeit eine Güterabwägung zwischen der Freiheit von Wissenschaft und beispielsweise dem Schutz von Persönlichkeitsrechten unumgänglich bleiben. Neben den Quellen in den Archiven sind die Militärfachzeitschriften der Bundeswehr ein bedeutender Ort der Überlieferung. Sie boten und bieten den Angehörigen der Streitkräfte ein wichtiges innermilitärisches Diskussionsforum. Als die langjährige Publikation »Truppenpraxis« Anfang der 2000er Jahre eingestellt wurde, versandete für den Historiker allerdings eine bedeutende Quelle über die innere Verfasstheit und die Stimmungslage in der Bundeswehr. ${ }^{6}$

Mit der Deutschen Einheit 1990 erweiterte sich die Forschung des damaligen MGFA um die Militärgeschichte der DDR. Die in den Gesamtzusammenhang der Geschichte des Kalten Krieges eingebettete Grundlagenforschung zur Militär- und Sicherheitspolitik sowie zu den bewaffneten Organen der DDR bildete den Beitrag der Militärhistoriker zur Aufarbeitung der Geschichte der SED-Diktatur. Die

5 Vgl. anders Christian Th. Müller, US-Truppen und Sowjetarmee in Deutschland. Erfahrungen, Beziehungen, Konflikte im Vergleich, Paderborn [u.a.] 2011 (= Krieg in der Geschichte, 70); in die Nachkriegszeit hineinreichend: Eckard Michels, Deutsche in der Fremdenlegion 1870-1965. Mythen und Realitäten, kartonierte Sonderausgabe, Paderborn [u.a.] 2011 (= Krieg in der Geschichte, 2).

6 Vgl. Deutsche Militärfachzeitschriften im 20. Jahrhundert. Im Auftrag des MGFA hrsg. von Markus Pöhlmann, Potsdam 2012 (= Potsdamer Schriften zur Militärgeschichte, 17). 
grundlegende Untersuchung der bewaffneten Macht, insbesondere des Militärs, als einer wichtigen Stütze des DDR-Regimes schuf eine entscheidende Voraussetzung dafür, wesentliche Funktionsmechanismen und Wechselwirkungen der Herrschaftssicherung der SED nach innen und außen zu begreifen. Das erste Forschungsprojekt zur ostdeutschen Militärgeschichte startete 1991. Es bildete die Basis für eine systematische Aufarbeitung der Militärgeschichte des ostdeutschen Staates von den Anfängen bis zu seinem Ende. 1998 erfolgte am MGFA die Bildung des eigenständigen Forschungsbereichs »Militärgeschichte der DDR im Bündnis«, dessen Forschungsleistungen der Wissenschaftsrat 2006 ein Alleinstellungsmerkmal zuerkannte.

In einem Aufsatz zum Stand und zur Zukunft der Militärgeschichte der DDR, der in dieser Zeitschrift vor zehn Jahren erschien, ${ }^{7}$ bilanzierten Wissenschaftler des MGFA die bis dahin erreichten Forschungsergebnisse zur Militärgeschichte der DDR und wiesen auf noch zu erforschende Themen hin. ${ }^{8}$ Seit der Wiedervereinigung Deutschlands sind etwa 7000 Publikationen zur Geschichte der DDR in Buchform erschienen. ${ }^{9}$ Die bereits früher getroffene grundsätzliche Feststellung, dass militärhistorische DDR-Forschung in der Öffentlichkeit und in der Wissenschaft zunehmend wahrgenommen und als unverzichtbarer Beitrag zur Aufarbeitung der SED-Diktatur verstanden wird, ${ }^{10}$ scheint prinzipiell weiter zuzutreffen. Ungebrochen ist das Bewusstsein der Militärhistoriker, einen zentralen Bestandteil der Gesellschaft der DDR zu erforschen, dessen Kenntnis für das Verstehen des SED-Staates als zweite deutsche Diktatur unerlässlich ist.

7 Vgl. Heiner Bröckermann, Torsten Diedrich, Winfried Heinemann, Matthias Rogg und Rüdiger Wenzke, Die Zukunft der DDR-Militärgeschichte. Gedanken zu Stand und Perspektiven der Forschung. In: Militärgeschichtliche Zeitschrift, 66 (2007) 1, S. 71-99.

8 Zwischenbilanzen zur militärhistorischen DDR-Forschung boten bisher drei Aufsätze aus den Jahren 2003, 2007 und 2011. Vgl. Hans Ehlert und Armin Wagner, Äußere Sicherheit und innere Ordnung. Armee, Polizei und paramilitärische Organisationen im SED-Staat. In: Bilanz und Perspektiven der DDR-Forschung. Hrsg. von Rainer Eppelmann, Bernd Faulenbach und Ulrich Mählert im Auftrag der Stiftung Aufarbeitung der SED-Diktatur, Paderborn [u.a.] 2003, S. 139-150; Bröckermann [u.a.], Die Zukunft der DDR-Militärgeschichte (wie Anm.7); Gerhard P. Groß, Zwanzig Jahre nach dem Mauerfall. Bilanz und Perspektive der wissenschaftlichen Forschung über die Nationale Volksarmee. In: Die NVA und die Ungarische Volksarmee im Warschauer Pakt. Im Auftrag des MGFA hrsg. von Hans-Hubertus Mack, Laszlo Veszpremy und Rüdiger Wenzke, Potsdam 2011 (= Potsdamer Schriften zur Militärgeschichte, 15), S. 21-30.

9 Ulrich Mählert, Totgesagte leben länger. Oder: Konjunkturen der DDR-Forschung vor und nach 1989. Eine Einführung. In: Die DDR als Chance. Neue Perspektiven auf ein altes Thema, Berlin 2016, S. 9.

10 So zuletzt auch Groß, Zwanzig Jahre (wie Anm. 8), S. 21 f. 
Neue militärgeschichtliche Forschungsergebnisse fanden inzwischen Aufnahme in grundlegende Überblickswerke zur DDR-Historie. ${ }^{11}$ Dennoch scheint die Insellage der Militärgeschichte der DDR - ähnlich wie die der Bundeswehrgeschichte - trotz mancher Fortschritte noch immer nicht überwunden. So spielt die Militärgeschichte in dem 2016 erschienenen Buch der Bundesstiftung zur Aufarbeitung der SED-Diktatur »Die DDR als Chance«, in dem sich bekannte Zeithistoriker zur Rolle ihrer Spezialdisziplinen und Arbeitsfelder äußern, keine Rolle. ${ }^{12}$ Dabei waren vor allem in den letzten Jahren weder die Forschungsergebnisse noch die Projekte der Militärhistoriker zu übersehen. So erhielt die 2001 eröffnete Reihe »Militärgeschichte der DDR« im vergangenen Jahrzehnt weiter Zuwachs. Tatsächlich erschienen von 2007 bis Ende 2016 zwölf neue Bände sowie mehrere Neuauflagen. Das MGFA/ZMSBw führte auch in den zurückliegenden Jahren zeitgeschichtlich relevante Konferenzen sowie Workshops durch und ist mit zahlreichen Kooperationen Teil der nationalen und internationalen Forschungslandschaft. Die Zusammenarbeit mit dem Deutsch-Russischen Museum in BerlinKarlshorst bei der Erstellung einer modernen elektronischen Geodatenbank zu den Standorten der sowjetischen Truppen in der DDR ist das jüngste Beispiel. ${ }^{13}$

Während die Geschichte von Bundeswehr und NVA mit den oben benannten Einschränkungen in wesentlichen Teilen dennoch als einigermaßen gut erforscht gelten kann, trifft dies auf die Geschichte der Bündnisse nur bedingt zu. ${ }^{14}$ Wie bei der Erforschung der westdeutschen Militärgeschichte bildet auch hier der besondere Aktenzugang eine hohe Schwelle. Die Geschichte von Bündnissen ist zudem immer eine Geschichte der (gedachten und durchgeplanten) Koalitionskriegführung. Damit ist sie mehr als jede nationale Militärgeschichtsschreibung stets die Geschichte außenpolitischer Aushandlungsprozesse, in deren Zentrum die jeweilige Bündnisführungsmacht und ihr Kriegsbild stehen. Sowohl der multilaterale Charakter als auch das hohe Maß an politischer Einflussnahme jenseits

11 Vgl. u.a. Klaus Schroeder, Der SED-Staat. Geschichte und Strukturen der DDR 1949-1990, Köln, Weimar, Wien 2013; Dierk Hoffmann, Von Ulbricht zu Honecker. Die Geschichte der DDR 1949-1989, Berlin 2013 (= Deutsche Geschichte im 20. Jahrhundert, 15).

12 Vgl. Die DDR als Chance (wie Anm. 9). Zu den Debatten um die Rolle und Bedeutung der "DDR-Aufarbeitung « zeigen sich im Wesentlichen nur altbekannte Themenfelder.

13 Vgl. <www.mgfa.de/html/zms_standorte_ziele_gssd.php> (letzter Zugriff 18.1.2017). In diesem Zusammenhang werden auch die ebenfalls seit 2005 frei zugänglichen elektronischen Standortdatenbanken des ZMSBw zur Nationalen Volksarmee und den Grenztruppen der DDR sowie zur Bundeswehr schrittweise grundlegend technisch modernisiert.

14 Eine kommentierte Auswahlbibliografie zum Kalten Krieg und zur Rolle von NATO und Warschauer Pakt findet sich bei Dieter Krüger, Am Abgrund? Das Zeitalter der Bündnisse: Nordatlantische Allianz und Warschauer Pakt 1947 bis 1991, Fulda 2013 (= Schriftenreihe Point Alpha, 1), S. 213-223. 
rein militärischer Operationen bedingt einen archivalisch multinationalen und zugleich multiperspektivischen Ansatz.

Die Geschichte der Bündnisse vollzieht sich auf einer strategischen Ebene und ist somit an der Schnittstelle von Politik und Militär anzusiedeln. Deshalb verwundert es nicht, dass die Entwicklungen von NATO und Warschauer Pakt eher mit den Mitteln einer Politik- denn einer Militärgeschichtsschreibung zu fassen sind. Ihre Geschichte wird zudem - im Gegensatz zur Geschichte der nationalen Streitkräfte - zunächst in der übergeordneten politischen Sphäre des Kalten Krieges eingeordnet, der in den Bilanzen der letzten Jahre als geostrategischer und ideologischer Konflikt charakterisiert wurde. ${ }^{15}$ John Lewis Gaddis, der einerseits das stabilisierende Moment der weltweiten geostrategischen Auseinandersetzung hervorhebt und zugleich die Verantwortung für den Ursprung des Konfliktes bei der Sowjetunion und Stalin sieht, schloss an seine Studien aus den 1990er Jahren jüngere Werke an. ${ }^{16}$ Nach Georges-Henri Soutous Buch von 2001, ${ }^{17}$ das die ideologischen Ursachen und die Risiken des Kalten Krieges stärker betont, bot zuletzt Bernd Stöver 2011 eine deutschsprachige Überblicksdarstellung an. ${ }^{18}$ Daneben veröffentlichte das Hamburger Institut für Sozialforschung weitere Studien. ${ }^{19}$ Auch das MGFA/ZMSBw legte mit seinen Arbeiten über militärische Operationsplanungen ${ }^{20}$ und dem vergleichenden Blick auf die beiden deutschen Staaten in ihren Bündnissen international konzipierte Forschungsergebnisse vor. ${ }^{21}$ Im März

15 Einen Literaturüberblick bietet Jost Dülffer, Ost-West-Konflikt und Globalisierung. Neue Forschungen zum Kalten Krieg. In: Mittelweg 36, 22 (2013), 4, S. 57-76.

16 Vgl. John Lewis Gaddis, The long Peace. Inquiries into the history of the Cold War, New York [u.a.] 1987; John Lewis Gaddis, We now know. Rethinking Cold War History, Oxford 1997; John Lewis Gaddis, The Cold War. A new History, New York 2005; John Lewis Gaddis, The Cold War, London 2006; John Lewis Gaddis, Der Kalte Krieg. Eine neue Geschichte, München 2007.

17 Vgl. Georges-Henri Soutou, La guerre de cinquante ans. Le conflit Est-Ouest 1943-1990, Paris 2001.

18 Vgl. Bernd Stöver, Der Kalte Krieg. 1947-1991. Geschichte eines radikalen Zeitalters, München 2011.

19 Vgl. Studien zum Kalten Krieg, 6 Bde, Hamburg 2006-2013.

20 Vgl. Die Alpen im Kalten Krieg. Historischer Raum, Strategie und Sicherheitspolitik. In Zusammenarbeit der Landesverteidigungsakademie Wien und des Militärgeschichtlichen Forschungsamtes, Potsdam, hrsg. von Dieter Krüger und Felix Schneider, München 2012 (= Beiträge zur Militärgeschichte, 71); Dieter Krüger, Brennender Enzian. Die Operationsplanung der NATO für Österreich und Norditalien 1951 bis 1960, Freiburg i.Br. 2010 (= Einzelschriften zur Militärgeschichte, 46); Krüger, Am Abgrund? (wie Anm. 14); Schlachtfeld Fulda Gap. Strategien und Operationspläne der Bündnisse im Kalten Krieg. Hrsg. von Dieter Krüger, Fulda 2014 (= Point Alpha, 2).

21 Vgl. Wege zur Wiedervereinigung. Die beiden deutschen Staaten in ihren Bündnissen 1970 bis 1990. Im Auftrag des ZMSBw, hrsg. von Oliver Bange und Bernd Lemke, München 2013 (= Beiträge 
2015 begann das Berliner Kolleg Kalter Krieg/Berlin Center for Cold War Studies als ein gemeinsames Projekt des Hamburger Instituts für Sozialforschung, des Instituts für Zeitgeschichte München-Berlin, der Bundesstiftung zur Aufarbeitung der SED-Diktatur und der Humboldt-Universität zu Berlin seine Arbeit, an der auch das ZMSBw beteiligt ist. ${ }^{22}$

Im Folgenden sollen der Forschungsstand in den Hauptforschungsfeldern zur deutschen Militärgeschichte von 1945 bis 1990 erläutert, neue Arbeiten vorgestellt, Desiderata offengelegt und Perspektiven der Forschung diskutiert werden.

\section{Militärgeschichte der Bundesrepublik Deutschland}

Die Periodisierung der Geschichte der westdeutschen und seit 1990 gesamtdeutschen Streitkräfte kann unter verschiedenen Gesichtspunkten vorgenommen werden. Am weitesten verbreitet ist die Einteilung nach Regierungskoalitionen in fünf Phasen: Demilitarisierung und erste Anfänge (1948-1956), Aufbauphase (1956/57-1966/69), Reform und Konsolidierung (1966/69-1982) und letztlich eine Phase der Reife (1982-1990),23 an die sich mit der Zäsur von 1990 eine Zeitgeschichtsschreibung zur Sicherheitspolitik anschließt. Diese versteht sich als »Neueste Militärgeschichte« an der Schnittstelle von Wissenschaft, Politik, Öffentlichkeit und Streitkräften. ${ }^{24}$ Aber auch die Darstellung und Differenzierung

zur Militärgeschichte, 75); Grenzen überwinden. Schleswig-Holstein, Dänemark \& die DDR. Hrsg. von Aaron Jessen, Elmar Moldenhauer und Karsten Biermann, Husum 2016; The Long Détente. Changing Concepts of Security and Cooperation in Europe, 1950s-1980s. Ed. by Oliver Bange und Poul Villaume, Budapest 2016.

22 Vgl. <www.berlinerkolleg.com/de/profil/zentrum-fuer-militaergeschichte-und-sozialwissenschaften-der-bundeswehr-zmsbw> (letzter Zugriff 18.1.2017).

23 Diese Phaseneinteilung vor dem Hintergrund der aktuellen Forschungsliteratur bei Rink, Die Bundeswehr 1950/55 bis 1989 (wie Anm. 3). Für die Phasen zwei und drei vgl. auch die vom MGFA begründete Reihe »Sicherheit und Streitkräfte der Bundesrepublik Deutschland«, bislang 12 Bde, München 2006-2014.

24 Vgl. die Reihe »Neueste Militärgeschichte. Analysen und Studien«, bislang 5 Bde, Freiburg i.Br. 2012-2015, darin u.a. Auftrag Auslandseinsatz. Neueste Militärgeschichte an der Schnittstelle von Geschichtswissenschaft, Politik, Öffentlichkeit und Streitkräften. Im Auftrag des MGFA hrsg. von Bernhard Chiari, Freiburg i.Br. 2012 (= Neueste Militärgeschichte. Analysen und Studien, 1); Auslandseinsätze der Bundeswehr. Wegweiser zur Geschichte. Im Auftrag des MGFA hrsg. von Bernhard Chiari und Magnus Pahl, Paderborn [u.a.] 2010; Loretana de Libero, Tod im Einsatz. Deutsche Soldaten in Afghanistan, Potsdam 2014. 
der Entwicklung, zum Beispiel auf der Basis der jeweils gültigen NATO-Strategien, führen für die entsprechenden Themen der Bundeswehrgeschichte zu wichtigen Perspektivwechseln. ${ }^{25}$

Die Geschichte der Bundeswehr wird momentan, zugespitzt, durch zwei Narrative geprägt: eine Stabilisierungsgeschichte und eine Liberalisierungsgeschichte. ${ }^{26}$ Zum einen betont die Stabilisierungsgeschichte die erfolgreiche Modernisierung vor allem in den 1950er Jahren. Sie betrachtet die Entwicklung der Bundeswehr als Teil einer westdeutschen Erfolgsgeschichte, indem sie Konrad Adenauers Junktim von Wiederbewaffnung und Erlangung staatlicher Souveränität herausstellt. Zum anderen nimmt die Liberalisierungsgeschichte die Bundeswehr als latente Gefahr für die politischen Errungenschaften von Demokratisierung und Pluralisierung wahr, insofern sie die Bundeswehr als eine grundsätzlich restaurative Größe interpretiert, in der das Gegensatzpaar von Reformern und Traditionalisten in einem ständigen Ringen miteinander die militärpolitische Ausrichtung der Streitkräfte bestimmten. Auf Seiten der Militärreformer finden sich vermeintlich jüngere und der Demokratie zugewandte Akteure wie Wolf Graf von Baudissin, ${ }^{27}$ die mit dem Konzept der Inneren Führung für die Einbindung der Bundeswehr in die demokratische Gesellschaft stehen, während auf Seiten der Traditionalisten Namen wie Reinhard Gehlen, Friedrich Foertsch oder Albert Schnez fallen. Sie vertreten die sogenannte Sui-generis-These, nach der sich das Militär selbst außerhalb der Gesellschaft stelle. Hier entsteht vorschnell das Bild der jungen Demokraten gegen die Restauratoren von Reichswehr und Wehrmacht.

Die jüngere Forschung verneint jedoch die Annahme, dass es in der Geschichte der Bundeswehr einen trennscharfen Dualismus zwischen Reformern und Traditionalisten gegeben hat. Sie zeigt, dass gerade ein Soldat wie Albert Schnez, der aufgrund der nach ihm benannten Studie als Personifikation eines Traditionalisten galt, als Inspekteur des Heeres bei seiner Einschätzung des Kriegsbildes ebenso progressive wie traditionelle Auffassungen vertrat, wenn er sich für einen begrenzten und gegen einen allgemeinen Nuklearkrieg aussprach. ${ }^{28}$

25 Vgl. Die Bundeswehr 1955 bis 2005 (wie Anm. 3), S. 401.

26 Vgl. Geppert, Die Bundeswehr in der Geschichtsschreibung (wie Anm. 4), S. 37-43.

27 Vgl. Wolf Graf von Baudissin 1907-1993. Modernisierer zwischen totalitärer Herrschaft und freiheitlicher Ordnung. Im Auftrag des MGFA hrsg. von Rudolf J. Schlaffer und Wolfgang Schmidt, München 2007.

28 Vgl. Florian Reichenberger, Der gedachte Krieg - Vom Wandel der Kriegsbilder in der Führung der Bundeswehr im Zeitalter des Ost-West-Konflikts (in Vorb.). 
Neben ersten politologischen Studien in den 1960er Jahren ${ }^{29}$ entwickelten sich später vor allem die durch das MGFA herausgegebenen Reihen »Militärgeschichte seit 1945« und »Anfänge westdeutscher Sicherheitspolitik« (AWS) zu Referenzwerken für die von 1948 bis 1956 dauernde Vor- und Frühphase bundesdeutscher Militärgeschichte. ${ }^{30}$ Hinzukommt das 2006 aufgelegte Editionsprojekt »Der Bundestagsausschuss für Verteidigung «, das mit seinem jüngsten Band bis in das Jahr 1956 vordringt. ${ }^{31}$ Den Bogen von den Anfängen zur Reform- und Konsolidierungsphase (1966/69-1982) bis letztlich in die Phase der Reife (1982-1990) schlägt die ebenfalls seit 2006 vom MGFA herausgegebene Reihe »Sicherheitspolitik und Streitkräfte der Bundesrepublik Deutschland «. ${ }^{32}$

Gleichwohl findet die Geschichte der Vor- und Frühphase der westdeutschen Streitkräfte unverändert das besondere Interesse der Forschung, nicht zuletzt weil die Entwicklung der bundesdeutschen Sicherheitspolitik auf das engste mit der alliierten Besatzungspolitik verbunden war und Brüche wie Kontinuitätslinien im Nachkrieg ${ }^{33}$ noch deutlicher zu Tage traten. ${ }^{34}$ Seit einigen Jahren besteht der Eindruck, dass vor allem die erste Phase der sogenannten Wiederbewaffnung durch die historische Forschung hinreichend differenziert betrachtet worden sei..$^{35}$ Jüngere Forschungen lassen jedoch daran zweifeln, dass der Ausgangspunkt der

29 Vgl. Gerhard Wettig, Entmilitarisierung und Wiederbewaffnung in Deutschland 1943-1955. Internationale Auseinandersetzungen um die Rolle der Deutschen in Europa, München 1967 (= Schriften des Forschungsinstituts der deutschen Gesellschaft für auswärtige Politik e.V., 25); Arnulf Baring, Außenpolitik in Adenauers Kanzlerdemokratie. Bonns Beitrag zur Europäischen Verteidigungsgemeinschaft, München, Wien 1969 (= Schriften des Forschungsinstituts der deutschen Gesellschaft für auswärtige Politik e.V., 28).

30 Vgl. die Reihe Militärgeschichte seit 1945, 11 Bde, Boppard am Rhein 1975-2000 und die Reihe Anfänge westdeutscher Sicherheitspolitik, 4 Bde, München, Wien 1982-1997.

31 Vgl. Der Bundestagsausschuss für Verteidigung, bisher 4 Bde, Düsseldorf 2006-2016, zuletzt Bd 4: Der Ausschuss für Fragen der europäischen Sicherheit Juli 1955 bis Januar 1956. Im Auftrag des ZMSBw hrsg. von Dorothee Hochstetter und Dieter H. Kollmer, Düsseldorf 2017 (in Vorb.).

32 Vgl. die Reihe Sicherheitspolitik und Streitkräfte der Bundesrepublik Deutschland, bisher 12 Bde, München, 2006-2014.

33 Vgl. Jörg Echternkamp, Soldaten im Nachkrieg. Historische Deutungskonflikte und westdeutsche Demokratisierung 1945-1955, München 2014 (= Beiträge zur Militärgeschichte, 76); vgl. Kriegsenden, Nachkriegsordnungen, Folgekonflikte. Wege aus dem Krieg im 19. und 20. Jahrhundert. Im Auftrag des MGFA hrsg. von Jörg Echternkamp, Freiburg i.Br. 2012; Nachkrieg in Deutschland. Hrsg. von Klaus Naumann, Hamburg 2001.

34 Vgl. die Forderungen von Bruno Thoß, Die Zeit der Weltkriege - Epochen als Erfahrungseinheit. In: Erster Weltkrieg - Zweiter Weltkrieg. Ein Vergleich. Krieg, Kriegserlebnis, Kriegserfahrung in Deutschland. Im Auftrag des MGFA hrsg. von Bruno Thoß und Hans-Erich Volkmann, Paderborn [u.a.] 2002, S. 8f.

35 Vgl. Rudolf J. Schlaffer, Anmerkungen zu 50 Jahren Bundeswehr: Soldat und Technik in der »totalen Verteidigung «. In: Militärgeschichtliche Zeitschrift, 64 (2005), 2, S. 487-502. 
bundesdeutschen »Wiederbewaffnung « in der Koreakrise im Juni 1950 liegt und die Entwicklung über die Gründung der Zentrale für Heimatdienst zur Himmeroder Denkschrift in das Amt Blank geführt hat. Sie sehen den Wiederaufbau westdeutscher Streitkräfte vielmehr in einer längerfristigen Kontinuität. ${ }^{36}$ Ausgehend von der Aufdeckung eines um 1950 im südwestdeutschen Raum gekaderten Armeekorps ehemaliger Wehrmachtangehöriger und ihrer Verbände, lassen sich intensiver, als das bislang in der AWS-Reihe der Fall war, Verbindungen zur Organisation Gehlen nachweisen, die bis in das Jahr 1947 zurückreichen. Bereits das erste Kabinett Adenauer verfügte demnach in der Person des Bundesbauministers Eberhard Wildermuth (1890-1952, FDP) über einen Schattenminister für Verteidigungsfragen, der - gestützt auf die Organisation Gehlen und ehemalige Wehrmachtgenerale - seit Herbst 1949 ein bundespolitisches Sicherheitskonzept erarbeitete, ${ }^{37}$ dessen erste Verschriftlichung im »Besprechungsplan vom 5. Januar 1950« seinen Niederschlag fand. Es kann somit als die bislang älteste Konzeption zur westdeutschen Wiederbewaffnung gelten. ${ }^{38}$ Damit findet eine Verschiebung des bisherigen Narrativs statt, das viel stärker die Kontinuitäten im Sinne eines Liberalkonservatismus ${ }^{39}$ deutet und der Debatte um die vermeintliche Stunde Null neue Aspekte hinzufügt. Demnach sind ideengeschichtliche wie organisationsgeschichtliche Kontinuitäten jenseits des Nationalsozialismus auch in der Zeit der Weimarer Republik zu suchen. Die Frühphase der Bundeswehr steht darüber hinaus mit der Geschichte des Militärischen Abschirmdienstes, ${ }^{40}$ des Kriegsbildes der Bundeswehrführung ${ }^{41}$ sowie der Institutionalisierung der

36 Hier und im Folgenden: Agilolf Keßelring, Die Organisation Gehlen und die Verteidigung Westdeutschlands. Alte Elitedivisionen und neue Militärstrukturen, 1949-1953, Marburg 2014 (= UHK/BND, Studie 3).

37 Agilolf Keßelring und Thorsten Loch, Himmerod war nicht der Anfang. Bundesminister Eberhard Wildermuth und die Anfänge westdeutscher Sicherheitspolitik. In: Militärgeschichtliche Zeitschrift, 74 (2015), 1/2, S. 60-96.

38 Agilolf Keßelring und Thorsten Loch, Der »Besprechungsplan« vom 5. Januar 1950. Gründungsdokument der Bundeswehr? Eine Dokumentation zu den Anfängen westdeutscher Sicherheitspolitik. In: Historisch-Politische Mitteilungen, 22 (2015), S. 199-229.

39 Als »Liberalkonservative« beschreibt Jens Hacke diejenigen Akteure nach 1945, die zwar auf die Vermittlung moralischer Werte zurückgriffen, dabei aber eine Mischung aus Traditionsbezug und Offenheit nutzten, um "modernitätsermöglichend " zwischen dem Bewahren und dem Erneuern auf der Basis konventioneller moralischer Grundsätze wirken zu können. Vgl. Jens Hacke, Die Bundesrepublik als Idee. Zur Legitimationsbedürftigkeit politischer Ordnung, Hamburg 2009, S. 102-106.

40 Vgl. hierzu künftig Helmut H. Hammerich, Geschichte des MAD 1956 bis 1990 (in Vorb., 2018). 41 Vgl. Reichenberger, Der gedachte Krieg (wie Anm. 28). 
historischen Bildung in den Streitkräften ${ }^{42}$ und ihrer Umsetzung ${ }^{43}$ weiterhin im Fokus.

Nachdem für die bundesdeutschen Verteidigungsminister, die gemäß Grundgesetz die Befehls- und Kommandogewalt innehatten, schon seit Längerem umfassende Biografien und Autobiografien vorliegen, ${ }^{44}$ beschäftigt sich die historische Forschung seit geraumer Zeit auch mit den Soldaten der Bundeswehr. Zunächst wurden zentrale Akteure der ersten Phase der Bundeswehrgeschichte näher betrachtet. ${ }^{45}$ Daran anknüpfend wurde in einem umfassenden Projekt die Aufbaugeneration exemplarisch und gruppenbiografisch erfasst und analysiert. ${ }^{46}$ Aber auch die Herkunft und Einstellungen der Militärelite der Bundeswehr sind generationsgeschichtlich ergründet worden. ${ }^{47}$

42 Vgl. hierzu das Dissertationsprojekt von Cornelia Grosse (Potsdam), Die Historische Bildung im Spannungsfeld von Bundeswehr und Gesellschaft (Arbeitstitel).

43 Vgl. »Vom Einsatz her denken!« Bedeutung und Nutzen von Militärgeschichte zu Beginn des 21. Jahrhunderts. Im Auftrag des ZMSBw hrsg. von Dieter H. Kollmer, Potsdam 2013 (= Potsdamer Schriften zur Militärgeschichte, 23).

44 Die Untersuchung der führenden Köpfe des Bundesministeriums der Verteidigung stellt ein Desiderat dar. $\mathrm{Zu}$ Theodor Blank existiert keine umfassende und wissenschaftlichen Ansprüchen genügende Biografie. Zu Franz Josef Strauß jüngst Horst Möller, Franz Josef Strauß. Herrscher und Rebell, München 2015; vgl. auch Volker Koop, Kai-Uwe von Hassel. Eine politische Biographie, Köln 2007; Dieter E. Kilian, Kai-Uwe von Hassel und seine Familie. Zwischen Ostsee und Ostafrika. Militär-biographisches Mosaik, Berlin 2013; Torsten Oppelland, Gerhard Schröder (1910-1989). Politik zwischen Staat, Partei und Konfession, Düsseldorf 2002; Claus Jander, Der Einfluss des Bundesministers der Verteidigung Georg Leber auf das innere Gefüge der Bundeswehr, Berlin 2006; Volker Rühe - in der Mitte Europas. Hrsg. durch das MGFA, Potsdam 2012. Exemplarisch über Helmut Schmidt vgl. u.a. Detlef Bald, Politik der Verantwortung. Das Beispiel Helmut Schmidt: Das Primat des Politischen über das Militärische 1965-1975, Berlin 2008.

45 Vgl. Karl Feldmeyer und Georg Meyer, Johann Adolf Graf von Kielmannsegg 1906-2006. Deutscher Patriot, Europäer, Atlantiker. Mit einer Bild- und Dokumentenauswahl von Helmut R. Hammerich, Hamburg, Berlin, Bonn 2007; Wolf Graf von Baudissin (wie Anm. 27); John Zimmermann, Ulrich de Maizière. General der Bonner Republik. 1912 bis 2006, München 2012; Dorothee Hochstetter, Hasso von Manteuffel (1897-1978). Vom Panzergeneral zum Parlamentarier der FDP. In: Jahrbuch zur Liberalismus-Forschung, 27 (2015), S. 205-237; vgl. zudem Dieter Krüger, Hans Speidel und Ernst Jünger: Freundschaft und Geschichtspolitik im Zeichen der Weltkriege, Paderborn [u.a.] 2016; Peter M. Quadflieg, Gerhard Graf von Schwerin (1899-1980). Wehrmachtgeneral, Kanzlerberater, Lobbyist, Paderborn [u.a.] 2016; für die Auseinandersetzung um die Rolle Schwerins vgl. Alaric Searle, Internecine Secret Service Wars Revisited. The Intelligence Career of Count Gerhard von Schwerin, 1945-1956. In: Militärgeschichtliche Zeitschrift, 71 (2012), 1, S. 25-55.

46 Vgl. Militärische Aufbaugenerationen der Bundeswehr 1955 bis 1970. Ausgewählte Biografien. Im Auftrag des MGFA hrsg. von Helmut R. Hammerich und Rudolf J. Schlaffer, München 2011 (= Sicherheitspolitik und Streitkräfte der Bundesrepublik Deutschland, 10).

47 Vgl. Klaus Naumann, Generale in der Demokratie. Generationsgeschichtliche Studien zur Bundeswehrelite, Hamburg 2007; Dieter E. Kilian, Führungseliten. Generale und Admirale der 
In diesem Zusammenhang schreitet die Forschung zur Geschichte der Bundeswehr für die Phasen der Konsolidierung (1966/69-1982) und der Reife (1982-1990) voran. Charakteristisch ist ihre thematische Vielfalt: Sie widmet sich der Einbettung der Bundeswehr in die jeweilige NATO-Strategie, ${ }^{48}$ den Bedingungen militärisch-operativer Planung, den grundlegenden Organisations- und Strukturgeschichten der Teilstreitkräfte und ihrer Truppengattungen. ${ }^{49}$ Neben diesen militärorganisatorischen Aspekten werden die innenpolitische Dimension, ${ }^{50}$ insbesondere die politische Kontrolle der Soldaten, ${ }^{51}$ und die Integration der Bundeswehr in die Gesellschaft erörtert. ${ }^{52}$ Kulturgeschichtliche Fragestellungen

Bundeswehr 1955-2015. Politische und militärische Führung, Bielefeld 2014; Clemens Range, Kriegsgedient. Die Generale und Admirale der Bundeswehr, Müllheim-Britzingen 2013.

48 Vgl. Bruno Thoß, NATO-Strategie und nationale Verteidigungsplanung. Planung und Aufbau der Bundeswehr unter den Bedingungen einer massiven atomaren Vergeltungsstrategie 1952 bis 1960, München 2006 (= Sicherheitspolitik und Streitkräfte der Bundesrepublik Deutschland, 1).

49 Vgl. Bernd Lemke, Dieter Krüger, Heinz Rebhan und Wolfgang Schmidt, Die Luftwaffe 1950 bis 1970. Konzeption, Aufbau, Integration, München 2006 (= Sicherheitspolitik und Streitkräfte der Bundesrepublik Deutschland, 2); Helmut R. Hammerich, Dieter H. Kollmer, Martin Rink und Rudolf Schlaffer, Das Heer 1950 bis 1970. Konzeption, Organisation und Aufstellung. Unter Mitarbeit von Michael Poppe, München 2006 (= Sicherheitspolitik und Streitkräfte der Bundesrepublik Deutschland, 3); Johannes Berthold Sander-Nagashima, Die Bundesmarine 1955 bis 1972. Konzeption und Aufbau. Mit Beiträgen von Rudolf Arendt, Sigurd Hess, Hans Joachim Mann und Klaus-Jürgen Steindorff, München 2006 (= Sicherheitspolitik und Streitkräfte der Bundesrepublik Deutschland, 4); André Deinhardt, Panzergrenadiere im Kalten Krieg. Die Geschichte einer Truppengattung zwischen »Massive Retaliation« und "Flexible Response« 1960 bis 1970, München 2012 (= Sicherheitspolitik und Streitkräfte der Bundesrepublik Deutschland, 11); zur Geschichte der Luftwaffe vgl. zudem die Schriften zur Geschichte der Deutschen Luftwaffe, bislang 4 Bde, Essen, Berlin 2010-2016; zur Bundesmarine vereinzelt in: Kleine Schriftenreihe zur Militär- und Marinegeschichte, bislang 25 Bde, Bochum 2001-2016; vgl. auch Deutsche Marine im Wandel. Vom Symbol nationaler Einheit zum Instrument internationaler Sicherheit. Im Auftrag des MGFA hrsg. von Werner Rahn, München 2005 (= Beiträge zur Militärgeschichte, 63).

50 Vgl. Alessandra Ferretti und Patrick Bernhard, Pazifismus per Gesetz? Krieg und Frieden in der westdeutschen Verfassungsdiskussion, 1945-1949. In: Militärgeschichtliche Zeitschrift, 66 (2007), 1, S. 45-69.

51 Vgl. Frank Nägler, Der gewollte Soldat und sein Wandel. Personelle Rüstung und Innere Führung in den Aufbaujahren der Bundeswehr 1956 bis 1964/65, München 2010 (= Sicherheitspolitik und Streitkräfte der Bundesrepublik Deutschland, 9); Rudolf J. Schlaffer, Der Wehrbeauftragte 1951 bis 1985. Aus Sorge um den Soldaten, München 2006 (= Sicherheitspolitik und Streitkräfte der Bundesrepublik Deutschland, 5).

52 Vgl. Esther-Julia Howell, Von den Besiegten lernen? Die kriegsgeschichtliche Kooperation der U.S. Armee und der ehemaligen Wehrmachtelite 1945-1961, Berlin 2016 (= Studien zur Zeitgeschichte, 90); Wolfgang Schmidt, Integration und Wandel. Die Infrastruktur der Streitkräfte als Faktor sozioökonomischer Modernisierung in der Bundesrepublik 1955 bis 1975, München 2006 (= Sicherheitspolitik und Streitkräfte der Bundesrepublik Deutschland, 6); Patrick Bernhard, 
ergänzen diese Perspektiven. ${ }^{53}$ Querschnittlich angelegte Betrachtungen von Themenfeldern wie Militär und Geheimdienst, ${ }^{54}$ Militär und Medien, ${ }^{55}$ Traditionen, ${ }^{56}$ militärischer Alltag, Kultur und Mentalitäten ${ }^{57}$ sowie soldatisches Selbstverständnis runden die Forschungen zur Geschichte der Bundeswehr bis in die 1970er Jahre - und zum Teil darüber hinaus $-a b .^{58}$

Kriegsdienstverweigerung per Postkarte: Ein gescheitertes Reformprojekt der sozialliberalen Koalition. 1969-1978. In: Vierteljahrshefte für Zeitgeschichte, 53 (2005), S. 109-139; Markus Gunkel, Der Kampf gegen die Remilitarisierung. Friedensbewegung in Hamburg 1950 bis 1955, Frankfurt a.M. 2009.

53 Vgl. Thorsten Loch, Das Gesicht der Bundeswehr. Kommunikationsstrategien in der Freiwilligenwerbung der Bundeswehr, München 2008 (= Sicherheitspolitik und Streitkräfte der Bundesrepublik Deutschland, 8).

54 Vgl. Spione und Nachrichtenhändler. Geheimdienstkarrieren in Deutschland 1939-1989. Hrsg. von Helmut Müller-Enbergs und Armin Wagner, Berlin 2016; Matthias Uhl und Armin Wagner, BND contra Sowjetarmee. Westdeutsche Militärspionage in der DDR, Berlin 2007 (= Militärgeschichte der DDR, 14); Achtung Spione. Geheimdienste in Deutschland von 1945 bis 1956. Hrsg. von Magnus Pahl, Georg Pieken und Matthias Rogg, 2 Bde, Dresden 2016 (= Forum MHM. Schriftenreihe des Militärhistorischen Museums der Bundeswehr, 11).

55 Vgl. Heiner Möllers, Die Kießling-Affaire 1984. Zur Rolle der Medien im Skandal um die Entlassung von General Dr. Günter Kießling. In: Vierteljahrshefte für Zeitgeschichte, 64 (2016), 3, S. 517-540; Robert G. Moeller, Kämpfen für den Frieden: 08/15 und westdeutsche Erinnerungen an den Zweiten Weltkrieg. In: Militärgeschichtliche Zeitschrift, 64 (2005), 2, S. 359-389; Loch, Das Gesicht der Bundeswehr (wie Anm. 53).

56 Vgl. Tradition für die Bundeswehr. Neue Aspekte einer alten Debatte. Hrsg. von Eberhard Birk, Winfried Heinemann und Sven Lange, Berlin 2011; Loretana de Libero, Tradition in Zeiten der Transformation: Zum Traditionsverständnis der Bundeswehr im frühen 21. Jahrhundert, Paderborn [u.a.] 2006; als Beispiel einer Truppengeschichte siehe Das Wachbataillon beim Bundesministerium der Verteidigung (1957-2007). Geschichte - Auftrag - Tradition. Im Auftrag des Wachbataillons beim Bundesministerium der Verteidigung in Zusammenarbeit mit dem MGFA hrsg. von Thorsten Loch, Hamburg 2007.

57 Vgl. Ariane Slater, Militärsprache. Die Sprachpraxis der Bundeswehr und ihre geschichtliche Entwicklung. Unter Mitwirkung von Arno Fischer, Freiburg i.Br. 2015 (=Einzelschriften zur Militärgeschichte, 49); Martin Kutz, Deutsche Soldaten. Eine Kultur- und Mentalitätsgeschichte, Darmstadt 2006; Loretana de Libero, Rache und Triumph. Krieg, Gefühle und Gedenken in der Moderne, München 2014 (= Beiträge zur Militärgeschichte, 73).

58 Vgl. Kriegsbrauch und berufliches Selbstverständnis des Soldaten. Im Auftrag des MGFA und des Zentrums für Transformation der Bundeswehr hrsg. von Martin Hofbauer und Raimond W. Wagner, Freiburg i.Br. 2012 (= Einzelschriften zur Militärgeschichte, 47). Eine zusätzliche, wissenschaftlich kritisch zu reflektierende Quelle bieten Erinnerungen und Berichte von Zeitzeugen. Vgl. dazu u.a. Blue Braun, Erinnerungen an die Marine 1956-1996, Berlin 2012; Harald Volkmar Schlieder, Kommando zurück! Erinnerungen, Berlin 2012; Klaus Grot, So war's damals. Dienstchronik eines Pionieroffiziers im Kalten Krieg 1954-1991, Berlin 2014; Rainer Buske, Eine Reise ins Innere der Bundeswehr. Wundersame Geschichten aus einer anderen Welt, Berlin 2016. 
Die Geschichte des Sanitätsdienstes der Bundeswehr hingegen ist bei Weitem nicht so gut erforscht, allerdings inzwischen auch kein unbeschriebenes Blatt mehr. In den vergangenen Jahrzehnten erschienen verschiedene Forschungsergebnisse zur Geschichte der Wehrmedizin - der Begriff wird hier wertneutral verwendet - seit dem Zweiten Weltkrieg. Allerdings waren diese Publikationen oft anlassbezogen oder setzten sich mit Einzelaspekten der Geschichte des Sanitätsdienstes der Bundeswehr auseinander, ohne dass der Anspruch auf den Versuch einer umfassenden und systematischen Aufarbeitung erhoben werden konnte. ${ }^{59}$ Eine Ausnahme bildet die Veröffentlichung von reichhaltigem Quellenmaterial zur frühen Entwicklung des Sanitätsdienstes der Bundeswehr bis $1965 .^{60}$

Für die Geschichtsschreibung des Sanitätsdienstes der Bundeswehr haben sich aus der Vielzahl der sanitätsdienstlich-wehrmedizinischen Fragestellungen zunächst drei thematische Schwerpunkte herauskristallisiert. Der erste Bereich betrifft die Geschichtskultur und das Geschichtsbewusstsein im Sanitätsdienst der Bundeswehr, d.h. die Frage nach den Traditionslinien und Traditionsvorstellungen sowie den Geschichtsbildern. Einen weiteren Schwerpunkt bildet das Themenfeld »berufliches Selbstverständnis im Sanitätsdienst«, das mit seinen unterschiedlichen Facetten, wie etwa der Spannung zwischen der ärztlichen Berufsausübung und militärischen Pflichten und Aufgaben oder Fragen zum militärärztlichen Alltag unter Einbeziehung ethischer Implikationen sowie des Berufs- und Standesrechts, als beispielhaft für die inhaltliche Überschneidung der drei Einzelbereiche Geschichte, Theorie und Ethik der Wehrmedizin gelten kann. ${ }^{61}$ Der dritte Bereich betrifft die vergleichend-kontrastive Betrachtung der Geschichte der Wehrmedizin und ihre Einbindung in eine deutsch-deutsche

59 Dies wurde z.B. auch bei der Erarbeitung eines Beitrags zum fünfzigjährigen Bestehen der Bundeswehr offenbar: Erhard Grunwald und Ralf Vollmuth, Der Sanitätsdienst - Entstehung und Entwicklungen. In: Entschieden für Frieden. 50 Jahre Bundeswehr. 1955 bis 2005. Im Auftrag des MGFA hrsg. von Klaus-Jürgen Bremm, Hans-Hubertus Mack und Martin Rink, Freiburg i.Br., Berlin 2005, S. 183-198. Zum überwiegenden Teil stammen die greifbaren Beiträge zur Geschichte des Sanitätsdienstes nicht von Historikern, sondern von Sanitätsoffizieren und ähnlichen mit der Thematik befassten Personengruppen.

60 Vgl. Ernst Rebentisch, Die Gesundheit der Soldaten. Dokumente zum Sanitäts- und Gesundheitswesen der Bundeswehr, Gräfelfing 1995.

61 Vgl. zum Spannungsfeld zwischen der ärztlichen Berufsausübung und militärischen Pflichten und Aufgaben etwa Ralf Vollmuth, Humanitarian law and the Medical Service in armed conflicts: Medical and ethical problems. In: From Venus to Mars? Provincial Reconstruction Teams and the European Military Experience in Afghanistan, 2001-2014. On behalf of Bundeswehr Centre of Military History and Social Sciences ed. by Bernhard Chiari in collaboration with Thijs Brocades Zaalberg, Nicola Labanca and Ben Schoenmaker, Freiburg i.Br. [u.a.] 2014 (= Neueste Militärgeschichte. Analysen und Studien, 3), S. 393-402. 
Militärgeschichte des Kalten Krieges von 1970 bis zur Wiedervereinigung. Von wenigen Studien zu Einzelaspekten des Themas abgesehen, steht die Forschung hier noch am Anfang. ${ }^{62}$

Neben der Außen- und Sicherheitspolitik, der Organisations- und Strukturgeschichte sowie der Wehrmedizin rücken seit wenigen Jahren rüstungspolitische und -wirtschaftliche Fragen immer mehr in den Mittelpunkt der Forschung zur Geschichte der Bundeswehr. ${ }^{63}$ Dabei geht es vor allem um multinationale Kooperation, Lobbyismus und die Zusammenarbeit von Politik, Militär und Wirtschaft. ${ }^{64}$ In diesem Kontext wurde auch die Diskussion über den sogenannten MilitärischIndustriellen Komplex wieder aufgegriffen. Neueste Studien weisen nach, dass diverse Staaten den Einfluss der großen, weltweit agierenden Rüstungskonzerne auf die eigene Beschaffungspolitik so weit wie möglich einschränken wollen, indem sie mit gezielter Gesetzgebung und einem vorgegebenen Handlungsrahmen den Wettbewerb zwischen den Anbietern fördern und lenken. In der Bundesrepublik Deutschland entwickelte sich ein solches System mit dem Aufbau der Bundeswehr. Diese Politik, die mit prozesspolitischen Instrumenten einen nachfrageorientierten Markt zur Strukturerhaltung entgegen seiner eigentlichen Dynamik beeinflusst, wird in Erweiterung der lenkungswirtschaftlichen Ausprägung des Begriffs »Staatsinterventionismus« als »Rüstungsinterventionismus« bezeichnet. ${ }^{65}$

62 Eine dieser wenigen Ausnahmen bildet etwa die Studie zur Militärpharmazie von Carsten Gerd Dirks, Militärpharmazie in Deutschland nach 1945. Bundeswehr und Nationale Volksarmee im Vergleich, Stuttgart 2001 (= Quellen und Studien zur Geschichte der Pharmazie, 79), der auch eine gelungene, wenngleich natürlich nicht erschöpfende Gegenüberstellung der beiden Gesamtsysteme bietet (S. 5-87).

63 Vgl. u.a. Dieter H. Kollmer, »Klotzen, nicht Kleckern!« Die materielle Aufrüstung des Heeres von den Anfängen bis Ende der sechziger Jahre. In: Das Heer 1950 bis 1970 (wie Anm. 49), S. 485-614; Christoph Grams, Transatlantische Rüstungskooperation: Bedingungsfaktoren und Strukturen im Wandel (1990-2005), Baden-Baden 2007.

64 Vgl. Florian Seiller, Rüstungsintegration. Frankreich, die Bundesrepublik und die Europäische Verteidigungsgemeinschaft 1950-1954, Berlin [u.a.] 2015 (= Entstehung und Probleme des Atlantischen Bündnisses, 9); Claas Siano, Der Starfighter und die Luftwaffe. Rüstung im Spannungsfeld von Politik, Wirtschaft und Militär, Berlin 2016; Quadflieg, Gerhard Graf von Schwerin (wie Anm. 45); Thomas Haslinger, Bundeswehr und Ausrüstung: Die Beschaffung der Fahrzeugfamilien des Kampfpanzers LEOPARD 1 und des Schützenpanzers MARDER in den 1960er Jahren im Spannungsfeld zwischen Politik, Bundeswehr und Rüstungsindustrie, Inauguraldissertation, München 2015 <https://edoc.ub.uni-muenchen.de/19709/1/Haslinger_Thomas.pdf> (letzter Zugriff 17.1.2017).

65 Vgl. Militärisch-Industrieller Komplex? Rüstung in Europa und Nordamerika nach dem Zweiten Weltkrieg. Im Auftrag des ZMSBw hrsg. von Dieter H. Kollmer, Freiburg i.Br., Berlin, Wien 2015. 
An der Schnittstelle zur jüngsten Militärgeschichte der Bundeswehr ergeben sich neue Themen. Mit der Erforschung der "Armee der Einheit ${ }^{66}$ und der politischen Prozesse im $»$ Kosovo-Krieg ${ }^{67}$ werden weitere Pflöcke für die Historisierung der 1990er Jahre eingeschlagen. Diese Arbeiten werden hoffentlich eine Antwort darauf geben, inwiefern für den Historiker Vorsicht geboten ist, sobald er mit den zeitgenössischen Begriffen wie »Armee der Einheit« oder »Armee im Einsatz« konfrontiert wird.

Vereinfacht gesprochen lässt sich die Geschichtsschreibung zur Bundeswehr seit 2005 in zwei Felder einteilen. Einerseits gibt es eine Forschung, die die ältere Historiografie von den Anfängen westdeutscher Sicherheitspolitik kontinuierlich fortschreibt. Sie ist mittlerweile bis in die 1970er und teilweise in die 1980er Jahre hinein vorgestoßen und zeichnet erste Umrisse eines Gesamtbildes. In diesem Sinne sieht sich die Forschung an einem Punkt, an dem erstes Handbuchwissen sowie Überblicksdarstellungen geboten werden können. ${ }^{68}$ Andererseits formiert sich eine jüngere Forschung, die angesichts der Zäsur des Zweiten Weltkrieges erneut die Vor- und Frühphase der Bundeswehr ins Auge fasst und dabei die Tragfähigkeit der Erkenntnisse der über 40 Jahre zurückliegenden Grundlagenforschung, insbesondere des MGFA, angesichts neuer Aktenfunde und Interpretationen auf den Prüfstand stellt.

Den Kern der bisherigen Untersuchungen zur Bundeswehr bildet eine Geschichtsschreibung, welche die Streitkräfte der Bundesrepublik Deutschland als innen- und gesellschaftspolitisches Projekt wahrnimmt. Doch trotz zahlreicher Tiefenbohrungen, deren Ertrag außer Frage steht, zeichnen sich größere Desiderate ab. Dies betrifft etwa die Entwicklung und Einbettung der Presse- und Öffentlichkeitsarbeit der Bundeswehr wie auch die sogenannte Bundeswehrreform unter Helmut Schmidt von 1969 bis 1974. Inwiefern wurde beispielsweise ein Regierungswechsel auf der Ebene des Bundesministeriums der Verteidigung inhaltlichprogrammatisch und personell vollzogen? Eine Geschichte des Bundesministeriums der Verteidigung steht ebenso aus wie die Erforschung seiner wichtigsten Akteure: der Minister, der Generalstabsoffiziere und der Beamten.

66 Vgl. u.a. Peter Heinze, Bundeswehr »erobert« Deutschlands Osten, Berlin 2010; hierzu u.a. in Vorb. Jochen Maurer (Potsdam), (K)eine Armee der Einheit? (Arbeitstitel) und Martin Rink (Potsdam), Zur Organisationsgeschichte der Bundeswehr. Streitkräftestrukturen für eine Sicherheitspolitik in neuen Dimensionen seit 1990 (Arbeitstitel).

67 Vgl. das Dissertationsprojekt von Hans-Peter Kriemann (Potsdam), Die Rolle der Auslandseinsätze der Bundeswehr für Deutschlands Weg zur europäischen Gestaltungsmacht (Arbeitstitel).

68 Vgl. u.a. Rink, Die Bundeswehr (wie Anm. 3). 
Wir wissen kaum etwas über die Bundeswehr als genuin militärische Institution. Die Forderungen nach einer Organisationsgeschichte im Allgemeinen wie auch nach einer Geschichte des Personalkörpers im Besonderen blieben bezeichnenderweise seit 2005 weitgehend ungehört. ${ }^{69}$ Hierzu zählt die bereits erwähnte Organisationsgeschichte der Bundeswehr, deren Spitzengliederung als ein Aspekt Gegenstand jüngst abgeschlossener Forschung ist. ${ }^{70}$ Aber auch die Führungsphilosophie und das militärische Denken ${ }^{71}$ sowie die personellen Kontinuitäten jenseits einer Ereignisgeschichte, die sich an biografischen Facetten orientiert, sind bisher noch nicht angemessen erforscht worden.

Wenig wissen wir zudem über die konkreten Planungen der Bundesregierung, der Bundeswehrführung und anderer Ressorts für den Verteidigungsfall. Wie entwickelten sich gesamtstaatliche sicherheitspolitische Konzepte, wie gestaltete sich das Zusammenspiel der verschiedenen Bundesministerien für den Verteidigungsfall zwischen 1949 und der Wiedervereinigung? Lassen sich aus der Analyse des Kriegsbildes die grundlegenden Strukturen, einschließlich der Personal- und Materialstrukturen, ableiten? Eine genaue Kenntnis der Mobilmachungspläne und der Rolle der Territorialverteidigung wäre hilfreich, um zu verstehen, wie eng verzahnt einerseits Kriegsbild, operatives Denken sowie Personal- und Materialstruktur waren und wie sich andererseits das Zusammenwirken mit weiteren Akteuren staatlicher Sicherheit gestaltete. Die teilmobile »Friedens-Bundeswehr« ist nur durch den geplanten Aufwuchs und Einsatz der voll mobilisierten »Kriegs-Bundeswehr« verständlich.

Unzureichend ist in diesem Zusammenhang unser Wissen über die Aushandlungsprozesse zwischen Bund und Ländern im Spannungsfeld von Verteidigungsvorbereitungen und Infrastrukturmaßnahmen. ${ }^{72}$ Auch fehlen uns noch die notwendigen Informationen, um die tieferen Zusammenhänge zwischen wachsenden technischen Möglichkeiten, konventionell-taktischen Innovationen und deren Auswirkungen auf strategischer und politischer Ebene zu erkennen. Dies berührt die Frage nach der Interoperabilität zwischen den Teilstreitkräften und ihren Verbündeten im Friedens- wie im Verteidigungsfall. Zudem böte sich ein

69 Vgl. Loch, Das Gesicht der Bundeswehr (wie Anm. 53), S. 331; Schlaffer, Anmerkungen (wie Anm. 35), S. 502.

70 Vgl. Rudolf J. Schlaffer, Die Spitzengliederung der deutschen Streitkräfte 1870/71 bis zur Gegenwart (in Vorb.).

71 Vgl. zur Frage der Kontinuität im »operativen Denken« nach 1945 bei Gerhard P. Groß, Mythos und Wirklichkeit. Geschichte des operativen Denkens im deutschen Heer von Moltke d. Ä. bis Heusinger, Paderborn [u.a.] 2012 (= Zeitalter der Weltkriege, 9), S. 275-310.

72 Vgl. das Dissertationsprojekt von Leonie Hieck (Potsdam), Die Bundeswehr in Schleswig-Holstein im Spannungsfeld von Bund und Land (Arbeitstitel). Bislang nur Schmidt, Integration und Wandel (wie Anm. 52). 
besserer Einblick in die Strukturen und in das Selbstverständnis des bundesdeutschen Militärs in einem möglichen Krieg.

\section{Bündnisgeschichte: NATO}

Stellt schon für eine nationale Geschichte des Militärs die Recherche in verschiedenen Archiven regelmäßig eine Herausforderung dar, so trifft dies umso mehr auf Forschungsvorhaben zur NATO zu, für die neben deutschen auch amerikanische, französische und britische Archive sowie nicht zuletzt das NATO-Archiv in Brüssel eingesehen werden müssen. Der um die Jahrtausendwende am MGFA entwickelte Ansatz, die NATO-Geschichte als »staatenübergreifende Integrationsforschung über das internationale Kooperationssystem NATO « nicht nur als eine Aneinanderreihung von nationalen Militärgeschichten, sondern als » satlantische Politikı in ihrem weltpolitischen Umfeld wie in ihren Innenbeziehungen « zu begreifen, ist nach wie vor zukunftsweisend. ${ }^{73}$ Diese Forschung an der Schnittstelle zwischen Politik und Militär ist aber methodisch, sprachlich, organisatorisch und finanziell besonders aufwendig und kann deshalb nur durch institutionell verankerte und internationale Zusammenarbeit geleistet werden.

Die bundesdeutsche Erforschung der Geschichte der NATO gründet daher wie die Historiografie zur Militärgeschichte der Bundesrepublik Deutschland in erster Linie auf der Forschungsleistung des MGFA. ${ }^{74}$ Die Grundlagenforschung zu den Anfängen westdeutscher Sicherheitspolitik schuf hierfür die Voraussetzung. Die Arbeiten an der NATO-Geschichte begannen, nachdem die ersten drei Bände der AWS-Reihe Ende der 1980er Jahre abgeschlossen waren. Es hatte sich gezeigt, dass die Geschichte der NATO als ein wesentlicher Teil der Vorgeschichte der Sicherheitspolitik der Bundesrepublik und der Bundeswehr zu verstehen ist, denn die nationalen Verteidigungsplanungen der Bundesrepublik können nicht ohne Einordnung in die NATO-Strategie verstanden werden. ${ }^{75}$ Die Reihe $» E n t s t e-$ hung und Probleme des Atlantischen Bündnisses (bis 1956)« ist somit komple-

73 Vgl. Norbert Wiggershaus, Zur Konzeption einer NATO-Geschichte. In: Nationale Außen- und Bündnispolitik der NATO-Mitgliedstaaten. Im Auftrag des MGFA hrsg. von Norbert Wiggershaus und Winfried Heinemann, München 2000 (= Entstehung und Probleme des Atlantischen Bündnisses bis 1956, 2), S. XII f.

74 Vgl. die Reihe »Entstehung und Probleme des Atlantischen Bündnisses« (bis 1956), bislang 10 Bde, 1998-2015.

75 Es ist daher bezeichnend, dass der erste Band der Reihe »Sicherheitspolitik und Streitkräfte der Bundesrepublik Deutschland « dies zum Thema hat. Vgl. Thoß, NATO-Strategie und nationale Verteidigungsplanung (wie Anm. 48). 
mentär zur abgeschlossenen AWS-Reihe entstanden. Dies erklärt auch, warum der Titel der »NATO-Reihe« mit der Konzentration auf die Zeit von 1949 bis 1956 zunächst eine zeitliche Beschränkung erhielt: Die NATO-Geschichte bis 1956 gilt als Vorgeschichte der Bundeswehr inklusive ihres Beitritts. Die ersten bis 2003 erschienenen sechs Bände der Reihe gaben die thematische Gliederung vor. Mit dem 2009 publizierten siebten Band, der auf breiter Quellenbasis die Gründung der Nordatlantischen Allianz untersucht, darf die Forschung zu diesem Aspekt als weitgehend abgeschlossen gelten. ${ }^{76}$

Jüngere Arbeiten wenden sich nicht nur dem Zentrum des Bündnisses, sondern auch seinen Flanken und damit dem Kontakt zu dem Systemgegner ${ }^{77}$ und den Neutralen ${ }^{78} \mathrm{zu}$ oder nehmen bündnisinterne Zerreißproben unter die Lupe. ${ }^{79}$ So sind neue, international anschlussfähige Forschungsfelder entstanden. Ältere Studien zur atlantischen und europäischen Integration werden durch die jüngste Forschung über Rüstungsintegration vertieft und um das ebenfalls für die Bundeswehr untersuchte Feld der militärischen Rüstung erweitert. ${ }^{80}$ In einem folgenden Band der »NATO-Reihe« wird die NATO als militärische Allianz thematisiert und ihre innere Funktionsweise am Beispiel der Allied Mobile Force, einer mobilen Eingreiftruppe zur militärischen Verstärkung der Flanken, dargelegt. ${ }^{81}$ Damit liegt die erste Publikation in dieser Reihe vor, die sich nicht mehr der Entstehungszeit des Bündnisses widmet und die Entstehungsgeschichte der Bundeswehr begleitet, sondern analog zur fortschreitenden Erforschung bundesdeutscher Militärgeschichte in die 1960er bis 1980er Jahre vorstößt. Darüber hinaus sucht sie die Schnittstelle zur jüngsten Militärgeschichte. ${ }^{82}$ Weitere Unter-

76 Vgl. Gero von Gersdorff, Die Gründung der Nordatlantischen Allianz, München 2009 (= Entstehung und Probleme des Atlantischen Bündnisses, 7).

77 Vgl. Periphery or Contact Zone? The NATO Flanks 1961 to 2013. Ed. by Bernd Lemke, Freiburg i.Br. 2015 (= Neueste Militärgeschichte. Analysen und Studien, 4); Grenzen überwinden (wie Anm. 21); vgl. auch Harald van Neß, Die Geschichte der Life-Oak-Organisation als Beispiel moderner Planung und Vorbereitung zur Krisenbewältigung (in Vorb.).

78 Vgl. Agilolf Keßelring, Die Nordatlantische Allianz und Finnland 1949-1961. Perzeptionsmuster und Politik im Kalten Krieg, München 2009 (= Entstehung und Probleme des Atlantischen Bündnisses, 8).

79 Vgl. Stephan M. Brenner, Die NATO als Akteur im griechisch-türkischen Konflikt 1954 bis 1989 (in Vorb.); Stephan M. Brenner, Die Achillesferse der NATO: Die Nordatlantische Allianz und der griechisch-türkische Binnenkonflikt 1952-1989. Ein Überblick zum Stand der Forschung. In: Militärgeschichtliche Zeitschrift, 71, (2012), 1, S. 108-128.

80 Vgl. Militärisch-Industrieller Komplex (wie Anm. 65); Seiller, Rüstungsintegration (wie Anm. 64).

81 Vgl. Bernd Lemke, Die Allied Mobile Force 1961 bis 2002, Berlin [u.a.] 2015 (= Entstehung und Probleme des Atlantischen Bündnisses, 10).

82 Vgl. Periphery or Contact Zone? (wie Anm. 77). 
suchungen betrachten bezeichnenderweise mit der Luftwaffe eine in besonderem Maße in die Struktur der NATO integrierte und strategisch verwendbare Teilstreitkraft, an deren Beispiel wechselseitige Aushandlungsprozesse verdeutlicht werden können. ${ }^{83}$ Andere Studien analysieren die parteipolitische Sicht auf das westliche Bündnis. ${ }^{84}$

Neben Arbeiten, die sich ausschließlich mit der NATO befassen, sind in den letzten Jahren - angesichts des zunächst verbesserten Zugangs zu russischen Archiven - Studien getreten, die anhand bedeutender historischer Ereignisse, wie der Kubakrise ${ }^{85}$ oder des NATO-Doppelbeschlusses, ${ }^{86}$ aber auch anhand der Kriegspläne $^{87}$ und der Rüstungsbemühungen innerhalb der Bündnisse, ${ }^{88}$ durch einen transfergeschichtlichen oder vergleichenden Ansatz Ost und West miteinander verbinden. ${ }^{89}$ In Hinblick auf die Untersuchungen zum NATO-Doppelbeschluss gelingt es darüber hinaus, innenpolitische Fragen, etwa nach der Rolle der Friedensbewegung, in eine strategiegeschichtliche Perspektive $\mathrm{zu}$ integrieren. ${ }^{90}$ Ins-

83 Vgl. Dieter Krüger, Nationaler Egoismus und gemeinsamer Bündniszweck. Das »NATO Air Defence Ground Environment Programme« (NADGE) 1959-1968. In: Militärgeschichtliche Zeitschrift, 64 (2005) 2, S. 333-358; Dieter Krüger, Schlachtfeld Bundesrepublik? Europa, die deutsche Luftwaffe und der Strategiewechsel der NATO 1958 bis 1968. In: Vierteljahrshefte für Zeitgeschichte, 56 (2008), S. 171-225.

84 Vgl. Jan Hansen, Abschied vom Kalten Krieg? Die Sozialdemokraten und der Nachrüstungsstreit (1977-1987), Berlin 2016 (= Schriftenreihe der Vierteljahreshefte für Zeitgeschichte, 112).

85 Vgl. Vor dem Abgrund. Die Streitkräfte der USA und der UdSSR sowie ihrer deutschen Bündnispartner in der Kubakrise. Hrsg. von Dimitrij N. Filippovych und Matthias Uhl, München 2005 (= Schriftenreihe der Vierteljahreshefte für Zeitgeschichte, Sondernummer); Bernd Greiner, Die Kuba-Krise. Die Welt an der Schwelle zum Atomkrieg, München 2010.

86 Vgl. Gerhard Wettig, Entspannung, Sicherheit und Ideologie in der sowjetischen Politik 1969 bis 1979. Zur Vorgeschichte des NATO-Doppelbeschlusses. In: Militärgeschichtliche Zeitschrift, 68 (2009), 1, S. 75-116; Gerhard Wettig, Die Sowjetunion in der Auseinandersetzung über den NATODoppelbeschluss 1979-1983. In: Vierteljahrshefte für Zeitgeschichte, 57 (2009), S. 217-259.

$87 \mathrm{Vgl}$. War Plans and Alliances in the Cold War. Threat perceptions in the East and West. Ed. by Vojtech Mastny, Sven G. Holtsmark and Andreas Wenger, London, New York 2006; Die Alpen im Kalten Krieg (wie Anm. 20); Krüger, Brennender Enzian (wie Anm. 20); Blueprints for Battle. Planning for War in Central Europe 1948-1968. Ed. by Jan Hoffenaar and Dieter Krüger, Lexington, KY 2012.

88 Vgl. Militärisch-Industrieller Komplex (wie Anm. 65).

89 Vgl. Wege zur Wiedervereinigung (wie Anm. 21); Krüger, Am Abgrund? (wie Anm. 14); Schlachtfeld Fulda Gap (wie Anm. 20).

90 Dazu u.a. mit durchaus unterschiedlichen Sichtweisen: Zweiter Kalter Krieg und Friedensbewegung. Der NATO-Doppelbeschluss in deutsch-deutscher und internationaler Perspektive. Hrsg. von Philipp Gassert, Tim Geiger und Hermann Wentker, München 2011 (= Schriftenreihe der Vierteljahreshefte für Zeitgeschichte, Sondernummer); Holger Nehring und Benjamin Ziemann, Führen alle Wege nach Moskau? Der NATO-Doppelbeschluss und die Friedensbewegung - eine 
gesamt bleibt festzuhalten, dass eine Geschichte der NATO, wie nicht anders $\mathrm{zu}$ vermuten, auch künftig nur in einem internationalen Forschungsrahmen geschrieben werden kann.

\section{Militärgeschichte der DDR}

Die Forschung zur »Militärgeschichte der DDR im Bündnis« ist im letzten Jahrzehnt gut vorangeschritten. ${ }^{91}$ Sie konnte erneut einen erheblichen Erkenntnisund Wissenszuwachs sowohl für die Wissenschaft als auch für die Öffentlichkeit erbringen. Dies trifft auf die Geschichte der Nationalen Volksarmee (NVA) in der Ulbricht-Ära in besonderem Maße zu. In der Studie »Ulbrichts Soldaten« von 2013 wurden die ostdeutschen Streitkräfte von 1956 bis 1971 erstmals umfassender analysiert und wissenschaftlich bewertet. ${ }^{92}$ Damit konnten die Arbeiten zu den Anfängen der geheimen Aufrüstung und zur Kasernierten Volkspolizei stringent fortgesetzt werden. ${ }^{93}$ Andere Untersuchungen über den Zeitabschnitt der 1950er und 1960er Jahre wandten sich zumeist militärischen Einzelproblemen sowie mili-

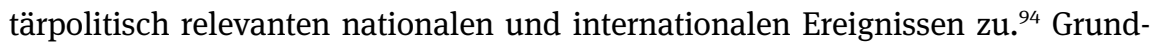

Kritik. In: Vierteljahrshefte für Zeitgeschichte, 59 (2011), S. 81-100; Gerhard Wettig, Der Kreml und die Friedensbewegung Anfang der achtziger Jahre, München 2012; »Entrüstet Euch!«. Nuklearkrise, NATO-Doppelbeschluss und Friedensbewegung. Hrsg. von Cristoph Becker-Schaum [u.a] unter Mitarb. von Laura Stapane, Paderborn [u.a.] 2012, engl. The Nuclear Crisis. The Arms Race, Cold War Anxiety and the German Peace Movement oft the 1980s. Ed. by Christoph BeckerSchaum, Philipp Gassert, Martin Klimke, Wilfried Mausbach and Marianne Zepp, New York 2016. 91 Die wertvolle Bibliografie von Hans Ehlert und Hans-Joachim Beth zur Militär- und Sicherheitspolitik in der SBZ/DDR endet 1995. Vgl. Die Militär- und Sicherheitspolitik in der SBZ/DDR. Eine Bibliographie. Im Auftrag des MGFA hrsg. von Hans Ehlert, bearb. von Hans-Joachim Beth, München 1996. Partielle Bibliografien zur NVA, die danach u.a. vom Bundesarchiv und von der Arbeitsgruppe Geschichte der NVA im Landesverband Ost des Deutschen Bundeswehrverbandes zusammengestellt wurden, bieten auch für die Zeit danach keinen umfassenden Überblick.

92 Vgl. Rüdiger Wenzke, Ulbrichts Soldaten. Die Nationale Volksarmee 1956 bis 1971. Hrsg. vom MGFA, Berlin 2013 (= Militärgeschichte der DDR, 22).

93 Vgl. Volksarmee schaffen - ohne Geschrei! Studien zu den Anfängen einer »verdeckten Aufrüstung « in der SBZ/DDR 1947 bis 1952. Im Auftrag des MGFA hrsg. von Bruno Thoß, München 1994 (= Beiträge zur Militärgeschichte, 51); Torsten Diedrich und Rüdiger Wenzke, Die getarnte Armee. Geschichte der Kasernierten Volkspolizei der DDR 1952 bis 1956. Hrsg. vom MGFA, 2. Aufl., Berlin 2003 (= Militärgeschichte der DDR, 1).

94 Vgl. u.a. Stefan Karner [u.a.], Prager Frühling. Das internationale Krisenjahr 1968. Beiträge, Weimar, Wien 2008 (= Veröffentlichungen des Ludwig Boltzman-Instituts für Kriegsfolgen-Forschung, Sonderbd 9/1); Korea - ein vergessener Krieg? Der militärische Konflikt auf der koreanischen Halbinsel 1950-1953 im internationalen Kontext. Hrsg. von Bernd Bonwetsch und Matthias Uhl, München 2012 (= Veröffentlichungen des Deutschen Historischen Instituts Moskau, 
legend neue, die bisherigen Forschungen revidierende Ergebnisse sind hier aller Voraussicht nach nicht mehr zu erwarten. Dennoch bleiben Desiderata. Diese betreffen in erster Linie die Wirtschafts- und Rüstungsproblematik, Struktur-, Ausbildungs- und Organisationsfragen in der Auf- und Ausbauphase der NVA sowie die Entwicklung der anderen bewaffneten Organe beim Aufbau der Landesverteidigung in der DDR.

In die Ära Honecker (1971-1989) konnten inzwischen ebenfalls wichtige Forschungsschneisen geschlagen werden. Das gilt für das Verhältnis von Armee und Gesellschaft, ${ }^{95}$ die grundlegenden Entwicklungen der Landesverteidigung und Militarisierung in der DDR, ${ }^{96}$ die SED-Militär- und Sicherheitspolitik, ${ }^{97}$ das widerständige Verhalten in der $\mathrm{NVA}^{98}$ und die Bausoldaten, ${ }^{99}$ das Wehrmachtserbe $^{100}$ und die Zivilverteidigung der DDR. ${ }^{101}$ Allerdings liegen auch hier noch Forschungsfelder brach, wie der massive Einsatz der NVA in der Volkswirtschaft, der Wandel des Feindbildes und des militärischen Denkens in den 1980er Jahren, der Alltag der Soldaten oder die Rolle von Frauen in der NVA. Zudem fehlt eine monografische Fortschreibung der DDR-Streitkräftegeschichte von 1971 bis 1989.

Schließlich zeigt auch die militärhistorische Aufarbeitung des letzten Zeitabschnitts der eigenständigen ostdeutschen Militärgeschichte, der vom Ende der 1980er Jahre bis zum 3. Oktober 1990 reicht, trotz einiger Fortschritte noch deutliche Lücken. ${ }^{102}$ So ist es nach wie vor dringend notwendig, das Wirken des gesamten Militär- und Sicherheitsapparates im Vorfeld und im direkten Prozess

3); Peter Joachim Lapp, Schüler in Uniform. Die Kadetten der Nationalen Volksarmee, Aachen 2009; Günther Glaser, Armee gegen das Volk? Zeitgenössische Studie mit Dokumenten zur Einsatzplanung des Militärs im Innern der DDR (1949-1965/66), Frankfurt a.M. 2009.

95 Vgl. Matthias Rogg, Armee des Volkes? Militär und Gesellschaft in der DDR. Hrsg. vom MGFA, Berlin 2008 (= Militärgeschichte der DDR, 15).

96 Vgl. Heiner Bröckermann, Landesverteidigung und Militarisierung. Militär- und Sicherheitspolitik der DDR in der Ära Honecker 1971-1989. Hrsg. vom MGFA, Berlin 2011 (= Militärgeschichte der DDR, 20).

97 Vgl. Oliver Bange, Sicherheit und Staat. Die Bündnis- und Militärpolitik der DDR im internationalen Kontext 1969 bis 1990, Berlin 2017 (= Militärgeschichte der DDR, 25).

98 Staatsfeinde in Uniform. Widerständiges Verhalten und politische Verfolgung in der NVA. Im Auftrag des MGFA hrsg. von Rüdiger Wenzke, Potsdam 2005 (= Militärgeschichte der DDR, 9).

99 Vgl. Bernd Eisenfeld und Peter Schicketanz, Bausoldaten in der DDR. Die »Zusammenführung feindlich-negativer Kräfte« in der NVA, Berlin 2011 (= Forschungen zur DDR-Geschichte).

100 Vgl. Daniel Niemetz, Das feldgraue Erbe. Die Wehrmachteinflüsse im Militär der SBZ/DDR. Hrsg. vom MGFA, Berlin 2006 (= Militärgeschichte der DDR, 13).

101 Vgl. Clemens Heitmann, Schützen und Helfen? Luftschutz und Zivilverteidigung in der DDR 1955 bis 1989/90, Berlin 2006 (= Militärgeschichte der DDR, 12).

102 Wege zur Wiedervereinigung (wie Anm. 21). 
des revolutionären Umbruchs 1989/90 zu analysieren. ${ }^{103}$ Darüber hinaus wird es Zeit, die »Nachwirkungen« der NVA, den Einfluss ihrer Angehörigen in der »Armee der Einheit« und der bundesdeutschen Gesellschaft interdisziplinär und zäsurübergreifend zu untersuchen. ${ }^{104}$

Insgesamt lassen sich in der militärhistorischen Forschung seit etwa 2005 einige bemerkenswerte Entwicklungen erkennen. So entstanden erstmalig - freilich mit unterschiedlicher Qualität - eigenständige Publikationen mit Überblickscharakter zur ostdeutschen Militärgeschichte, speziell zur Geschichte der NVA. Der zusammenhängende Blick auf die Zeit von 1945 bis 1990 lässt Besonderheiten, Kontinuitäten und Diskontinuitäten, Entwicklungsstränge, Prozesse und Ereignisse sichtbar werden. Er hilft, den Platz des DDR-Militärs in der deutschen Militärgeschichte des 20. Jahrhunderts näher zu bestimmen. Eine wissenschaftsfundierte und quellengestützte Gesamtdarstellung der »Militärgeschichte der SBZ/DDR « bleibt allerdings weiterhin ein Desiderat. ${ }^{105}$

Aber nicht nur die ersten Überblicksdarstellungen bildeten ein Novum in der militärgeschichtlichen Aufarbeitung der letzten Jahre. Auch die Erschließung neuer Themenfelder erreichte zum Teil eine neue Dimension. Beispielhaft zeigen dies vor allem Forschungen zum »Armeeknast« in Schwedt an der Oder. Selbst nach 1990 gab es zu diesem berüchtigten Militärgefängnis der NVA über zwei Jahrzehnte hinweg kaum Forschungen und Veröffentlichungen. Das Thema galt aufgrund der schlechten Quellenlage als nicht bearbeitbar. Die erste wissenschaft-

103 Zwar liegen hierzu einige wichtige Arbeiten vor, eine integrierte Gesamtdarstellung steht noch aus. Vgl. u.a. 1989 und die Rolle der Gewalt. Hrsg. von Martin Sabrow, Göttingen 2012; Mittendrin. Die Berliner Volkspolizei 1989/90. Hrsg. von Karl-Heinz Kriz und Hans-Jürgen Gräfe, Berlin 2014; "Damit hatten wir die Initiative verloren«. Zur Rolle der bewaffneten Kräfte in der DDR 1989/90. Im Auftrag des ZMSBw hrsg. von Rüdiger Wenzke, Berlin 2014 (= Militärgeschichte, 23).

104 Einen Anfang bildet u.a. 25 Jahre Armee der Einheit. Die »Geo-Dienste« im Zuge der Wiedervereinigung. Zeitzeugen berichten, Bd 1. Hrsg. vom Leiter Geoinformationsdienst der Bundeswehr, Euskirchen 2015 (= Geoinformationsdienst der Bundeswehr - Schriftenreihe, 2). Aus soziologischer Sicht vgl. u.a. Nina Leonhard, Integration und Gedächtnis. NVA-Offiziere im vereinigten Deutschland, Konstanz und München 2016.

105 Vgl. Guntram König, Das große Buch der Nationalen Volksarmee. Geschichte - Aufgaben Ausrüstung, Berlin 2008; Die Zeit nach 1945 (wie Anm. 3); Klaus Froh, Chronik der NVA, der Grenztruppen und der Zivilverteidigung der DDR 1956-1990, Berlin 2010; Winfried Heinemann, Die DDR und ihr Militär, München 2011 (= Militärgeschichte kompakt, 3); Marco Metzler, Nationale Volksarmee. Militärpolitik und politisches Militär in sozialistischer Verteidigungskoalition 1955/56 bis 1989/90, Baden-Baden 2012; Rüdiger Wenzke, Geschichte der Nationalen Volkarmee 1956-1990, Erfurt 2013; <www.bpb.de/politik/grundfragen/deutsche-verteidigungspolitik/2237 87/militaer-der-ddr> (letzter Zugriff 18.1.2017); Rüdiger Wenzke, Nationale Volksarmee. Die Geschichte, München 2014. 
liche Studie in Buchform erschien 2011. ${ }^{106}$ Erst auf dieser Grundlage entstanden Dokumentationen, Zeitzeugenberichte, weitere wissenschaftliche Publikationen und eine Ausstellung zum DDR-Militärstrafvollzug. ${ }^{107}$ Der in der DDR wurzelnde »Mythos Schwedt«, der sich die Unkenntnis der NVA-Soldaten über die realen Verhältnisse in der berüchtigten Disziplinareinheit zunutze machte und gezielt von den Vorgesetzten zur Erzeugung von Furcht und Anpassung in der Truppe instrumentalisiert wurde, konnte dadurch entzaubert werden.

Ebenso wurde inzwischen begonnen, andere wenig bekannte und zu DDRZeiten tabuisierte Themen $\mathrm{zu}$ bearbeiten. Dazu zählen die Militärbeziehungen und Militärhilfen der DDR in die »Dritte Welt«, über die Klaus Storkmann 2012 eine viel beachtete wissenschaftliche Studie vorgelegt hat. ${ }^{108}$ Der Autor räumt darin mit Legenden auf und bietet detaillierte Einsichten in die Ausbildung ausländischer Militärs in der NVA sowie in den Komplex der Waffenlieferungen der DDR in Staaten, die nicht dem Warschauer Pakt angehörten. Julian-André Finke beschreibt den Platz der ostdeutschen Luftstreitkräfte in den militärischen Planungen und ihre Integration in das Luftverteidigungssystem des Warschauer Paktes. Er macht deutlich, in welchem Maße die Führung der DDR durch sowjetische Vorgaben fremdbestimmt war. ${ }^{109}$

Eine überdurchschnittlich quantitative Erweiterung erfuhren im zurückliegenden Jahrzehnt vor allem Publikationen von ehemaligen NVA-Angehörigen zu verschiedenen Teilaspekten der NVA und zu den Grenztruppen. Dazu gehören übergreifende Darstellungen zur NVA-Geschichte, ${ }^{110}$ Publikationen zur Entwick-

106 Vgl. Rüdiger Wenzke, Ab nach Schwedt! Die Geschichte des DDR-Militärstrafvollzugs, 3. Aufl., Berlin 2016.

107 Vgl. Falk Bersch und Hans Hermann Dirksen, Strafvollzug Berndshof/Ueckermünde (1952-1972), Schwerin 2012; Spür die Angst, Die Disziplinarstrafeinheit des Militärgefängnisses in Schwedt/Oder. Zeitzeugen brechen ihr Schweigen. Hrsg. von Paul Brauhnert und Ilja Hübner, [o. O.] 2012; Torsten Dressler, »Stillgestanden - Blick zur Flamme!«. Das DDR-Militärstrafgefängnis und die NVA-Disziplinareinheit in Schwedt/Oder von 1968 bis 1990, Berlin, Bonn 2013; Der DDR-Militärstrafvollzug und die Disziplinareinheit in Schwedt (1968-1990). Zeitzeugen brechen ihr Schweigen. Hrsg. von Paul Brauhnert, Ilja Hübner und Arno Polzin, Berlin 2013; Helmut Irmen, Stasi und DDR-Militärjustiz. Der Einfluss des Ministeriums für Staatssicherheit auf Strafverfahren und Strafvollzug in der Militärjustiz der DDR, Berlin, Boston 2014; NVA-Soldaten hinter Gittern. Der Militärknast Schwedt als Ort der Repression, Sonderausstellung des Stadtmuseums Schwedt, Schwedt 2016.

108 Vgl. Klaus Storkmann, Geheime Solidarität. Militärbeziehungen und Militärhilfen der DDR in die »Dritte Welt«. Hrsg. vom MGFA, Berlin 2012 (= Militärgeschichte der DDR, 21).

109 Vgl. Julian-André Finke, Hüter des Luftraumes? Die Luftstreitkräfte der DDR im Diensthabenden System des Warschauer Paktes, Berlin 2010 (= Militärgeschichte der DDR, 18).

110 Vgl. dazu u.a. Fritz Minow, Die NVA und Volksmarine in den Vereinten Streitkräften. Geheimnisse der Warschauer Vertragsorganisation, Friedland 2011; Udo Beßer, Das Militärerho- 
lung von Ausbildungseinrichtungen, Truppenteilen, Verbänden, von Waffengattungen und Diensten ${ }^{111}$ sowie nicht zuletzt der Militärtechnik ${ }^{112}$ und dem Medizinischen Dienst der NVA. ${ }^{113}$ Obgleich diese Veröffentlichungen zumeist ein interessantes Detailwissen und mitunter auch sachbezogene historische Hintergrundinformationen enthalten, sind sie für die wissenschaftliche Aufarbeitung der DDR-Militärgeschichte nur bedingt von Nutzen. Dies ist vor allem der Quellen- und Methodenproblematik sowie der mitunter einseitigen politischen Färbung der Darstellungen geschuldet. Ausnahmen bestätigen auch hier die Regel. Dazu gehört zweifellos die 2011 erschienene Publikation zur Nutzung der militärischen Standorte im Osten Deutschlands. ${ }^{114}$ Es handelt sich dabei um eine sehr informative, in dieser Form einmalige Zusammenstellung von mehr als 600 ostdeutschen Standorten und deren Belegung durch Stäbe, Truppen, Depots und andere Einrichtungen. Ebenso haben sich ehemalige Offiziere der NVA bemüht, dem Leser mit dem Buch »Raketentruppen der NVA-Landstreitkräfte « ${ }^{115}$ einen durchaus sachlichen Einblick in eine geheimnisumwitterte Waffengattung der ostdeutschen Streitkräfte zu vermitteln. Ihnen ist es dabei neben allerlei technischen Beschreibungen gelungen, auf relevante historische Entwicklungen aufmerksam zu machen.

lungswesen in der DDR. Erholungsheime, Ferienlager, Kureinrichtungen, Berlin 2012; Bernd Biedermann und Hans-Georg Löffler, Militärs der DDR im Auslandsstudium. Erlebnisberichte Dokumente - Fakten, Berlin 2012.

111 Vgl. dazu u.a. Offiziershochschule der Luftstreitkräfte/Luftverteidigung »Franz Mehring«. Geschichte und Geschichten, Kamenz 2011; Peter Schreiber, Die Henne-Kaserne in Erfurt 1950 bis 1990, Bad Langensalza 2015; Bernd Tuchel und Rolf Zander, Das Panzerregiment-1 der NVA. Seine Geschichte und Erinnerungen, Berlin 2014; Knut Schäfer und Peter Seemann, DDR-Marine 1949-1990, Stuttgart 2014.

112 Vgl. dazu u.a. Dieter Flohr, Flugzeuge und Hubschrauber der NVA, München 2016; Andy Gröning, MiG-29, Stuttgart 2015; Dieter Flohr und Dirk Krüger, Die Panzer der Nationalen Volksarmee, München 2015; Lutz-Rainer Gau und Jörg Siegert, Radfahrzeuge der NVA. LKW, PKW und Kräder, Stuttgart 2014; Ulf Kaack, Die Schiffe der Volksmarine 1960-1990. Typenatlas NVA, München 2013.

113 Die wissenschaftliche Erforschung der Geschichte des Medizinischen Dienstes der NVA steckt auch nach zwei Sammelbänden noch in den Kinderschuhen. Vgl. dazu Der Medizinische Dienst der NVA - Teil I. Referate anlässlich des Workshops des Arbeitskreises Geschichte der Wehrmedizin vom 20.-21.04.2004 in Leipzig. Hrsg. von Franz-J. Lemmens und Wolfgang G. Locher, Klitzschen [2006] (= Beiträge Wehrmedizin und Wehrpharmazie, 17); Geschichte des Medizinischen Dienstes der NVA - Teil II. Hrsg. von Franz-J. Lemmens, Bonn 2009 (= Beiträge Wehrmedizin und Wehrpharmazie, 18).

114 Vgl. Olaf Kersten, Hans-Georg Löffler, Reinhard Parchmann und Siegfried Stoof, Garnisonen der NVA und der GSTD. Zur Nutzung der militärischen Standorte von 1871 bis 2010, Berlin 2011.

115 Vgl. Raketentruppen der NVA-Landstreitkräfte - Geheimhaltungsgrad aufgehoben. Hrsg. von Roland Großer, Halle 2012. 
Nicht zuletzt gab es bei der Erforschung des DDR-Grenzregimes und der Grenztruppen in den vergangenen Jahren Fortschritte. Zu nennen sind hier zuvorderst die unverzichtbaren Forschungen und Darstellungen von Peter Joachim Lapp. ${ }^{116}$ Neues Material und neue wissenschaftliche Sichtweisen finden sich zudem mit Blick auf den Dienst und den Alltag der Grenzpolizisten und -soldaten bei Gerhard Sälter und Jochen Maurer. ${ }^{117}$ Nach wie vor nehmen Publikationen zum Mauerbau und zum Mauerfall einen wichtigen Platz in der wissenschaftlichen und geschichtspolitischen Auseinandersetzung ein. Vor allem die Stiftung Berliner Mauer sowie Hans-Hermann Hertle (Zentrum für Zeithistorische Forschung Potsdam) haben sich hier große Verdienste erworben. ${ }^{118}$ Ehemalige Angehörige der NVA und der Grenztruppen äußerten sich ebenfalls zur historischen Problematik des Mauerbaus. ${ }^{119}$

Einen Aufschwung erlebte darüber hinaus die sogenannte Erinnerungsliteratur. Mannschaftssoldaten, Unteroffiziere, Offiziere und Generale/Admirale berichten zumeist autobiografisch über ihren Dienst in der NVA und bieten dem Leser zweifellos ein breites Spektrum von subjektiven Wahrnehmungen. ${ }^{120}$ Inzwischen liegen weit über 50 Einzelpublikationen dieses Genres vor, wobei die Anzahl jener Autoren überwiegt, die als Grundwehrdienstleistende

116 Vgl. Peter Joachim Lapp, Grenzregime der DDR, Aachen 2013; Jürgen Ritter und Peter Joachim Lapp, Deutschland grenzenlos. Bilder der deutschen-deutschen Grenze. Damals und heute, Berlin 2014; Peter Joachim Lapp, Die Mauer - eine Grenze durch Deutschland, Erfurt 2011.

117 Vgl. Gerhard Sälter, Grenzpolizisten. Konformität, Verweigerung und Repression in der Grenzpolizei und den Grenztruppen der DDR 1952 bis 1965. Hrsg. vom MGFA, Berlin 2009 (= Militärgeschichte der DDR, 17); Jochen Maurer, Dienst an der Mauer. Der Alltag der Grenztruppen rund um Berlin, Berlin 2011 (= Beiträge zur Geschichte von Mauer und Flucht); Jochen Maurer, Halt - Staatsgrenze! Alltag, Dienst und Innenansichten der Grenztruppen der DDR. Hrsg. vom ZMSBw, Berlin 2015 (= Militärgeschichte der DDR, 24).

118 Vgl. dazu u.a. Axel Klausmeier, Hinter der Mauer. Zur militärischen und baulichen Infrastruktur des Grenzkommandos Mitte, Berlin 2012; Hans-Hermann Hertle, Die Berliner Mauer: Biografie eines Bauwerkes, 2., aktual. Aufl., Berlin 2015; Hans-Hermann Hertle, Maria Nooke [u.a.], Die Todesopfer an der Berliner Mauer 1961-1989. Ein biographisches Handbuch. Hrsg. vom Zentrum für Zeithistorische Forschung Potsdam und der Stiftung Berliner Mauer, 2., durchges. Aufl., Berlin 2009; Hans-Hermann Hertle, The Berlin Wall Story. Biography of a Monument, 2nd ed., Berlin 2016; »Mit den Autos kommt die Ideologie«. Der Grenzübergang Helmstedt-Marienborn im Kontext der Teilung Deutschlands und Europas. Hrsg. von Sascha Möbius, Halle 2016.

119 Vgl. Heinz Keßler und Fritz Streletz, Ohne die Mauer hätte es Krieg gegeben. Zwei Zeitzeugen erinnern sich, Berlin 2011.

120 Erinnerungen, Zeitzeugenberichte und andere autobiografische Einträge von ehemaligen Angehörigen der NVA und der Grenztruppen finden sich zudem auf den einschlägigen Webseiten und in Internetportalen wie <www.nva-forum.de $>$. Darauf wird an dieser Stelle nicht weiter eingegangen. 
und Unteroffiziere in der NVA und den Grenztruppen gedient haben. ${ }^{121}$ Immerhin haben seit 2005 auch weitere Generale der NVA ihre Erinnerungen publiziert. $^{122}$

In Einzelstudien sind Forschungen vor allem zu Fragen der Wirtschaft, der Rüstung und Mobilmachung, zur Geschichte anderer bewaffneter Organe der DDR-Landesverteidigung einschließlich der sowjetischen Streitkräfte in der DDR, zur Geschichte der Teilstreitkräfte der NVA sowie zur Rolle von prägenden Persönlichkeiten der ostdeutschen Militärgeschichte zu beginnen oder fortzuführen. $\mathrm{Zu}$ prüfen ist auch, ob es beispielsweise mittels einer wissenschaftlichen Divisionsgeschichte möglich ist, die realen inneren Verhältnisse in der NVA, einschließlich des Soldatenalltags, mit einem deutlichen Erkenntnisgewinn zu untersuchen. ${ }^{123}$ Die Erinnerungsliteratur, die als Quelle insgesamt mit einem kritischen Blick zu betrachten ist, kann zweifellos helfen, die alltagsgeschichtlichen Forschungen zur Militärgeschichte der DDR um einen erinnerungskulturellen Aspekt zu erweitern. Bisher fehlen allerdings einschlägige Arbeiten, welche die Erinnerungen mit dem neuesten Stand der militärhistorischen Literatur abgleichen und quellenkritisch analysieren.

121 Hervorhebenswert sind u.a. Klaus Auerswald, ... sonst kommst du nach Schwedt! Bericht eines Militärstrafgefangenen, Rudolstadt 2010; Eckhard Ullrich, Kulturschock NVA. Briefe eines Wehrpflichtigen 1971-1973, Berlin 2013; Lutz Wolter, Wehrdienst bei der NVA. Erinnerungen an eine nicht nur, aber meistens, unangenehme Zeit, Norderstedt 2015; Richard Hebstreit, Grenzsoldat, Berlin 2007; Günter Hagemann, Gedient in Prora. Erinnerungen an meinen NVA-Wehrdienst, Berlin 2014.

122 Vgl. Horst Stechbarth, Soldat im Osten. Erinnerungen und Erlebnisse aus fünf Jahrzehnten, Hüllhorst 2006; Klaus-Dieter Baumgarten, Erinnerungen. Autobiographie des Chefs der Grenztruppen der DDR, Berlin 2008; Johannes Oreschko, Im Kalten Krieg. Ein General der Nationalen Volksarmee erinnert sich, Berlin 2011; Günther Sarge, Im Dienste des Rechts. Der oberste Richter der DDR erinnert sich; Berlin 2013. Zu den Erinnerungen von Offizieren siehe u. a. Rainer Langener, Meine Jahre auf dem Schleudersitz. Erinnerungen und Gedanken eines Militärfliegers der DDR, Aachen 2012; Michael Dumaschefski, Pier 4. Erinnerungen eines ehemaligen ostdeutschen Schnellbootskommandanten, Engelsdorf 2010; Wolfgang Herzig, Offizier im Chemischen Dienst der NVA. Eine etwas andere Lebensbetrachtung, Berlin 2015; Bernd Biedermann, Vom Kanonier zum Militärattaché der DDR. Eine Laufbahn im Kalten Krieg, Aachen 2015; Manfred Skeries, MiG29-Pilot in NVA und Bundeswehr. »So war das eben«. Ein ehemaliger Jagdflieger berichtet, Berlin 2015.

123 Bisher vorliegende Publikationen sind dazu nur bedingt geeignet. Siehe u.a. Klaus Froh, Zur Geschichte der 8. Mot. Schützendivision 1956 bis 1990, Schwerin 2006 (= Schriften zur Geschichte Mecklenburgs, 16); Klaus Froh, Die 1. MSD der NVA. Zur Geschichte der 1. Mot.-Schützendivision 1956-1990, Aachen 2017. 


\section{Bündnisgeschichte: Warschauer Pakt}

Die ostdeutsche Militärgeschichte ist eng verknüpft mit der Geschichte der sowjetischen Streitkräfte und des Warschauer Paktes. Vor diesem Hintergrund rückte die wissenschaftliche Beschäftigung mit operativen Fragen der Kriegsvorbereitung und Kriegführung in den vergangenen Jahren immer weiter in den Fokus der internationalen Forschung. Pionierarbeit leisteten auf diesem Feld anfangs vor allem das von der Eidgenössischen Technischen Hochschule in Zürich (ETHZ) betriebene "Parallel History Project on NATO and the Warsaw Pact ${ }^{124}$ und das vom Washingtoner Woodrow Wilson International Center verantwortete »Cold War International History Project «. ${ }^{125}$ Nunmehr sind auch in Deutschland bemerkenswerte Publikationen dazu erschienen. Hervorzuheben ist vor allem die 2013 am MGFA/ZMSBw veröffentlichte Studie »Kriegsschauplatz Deutschland». Ihr Autor Siegfried Lautsch war in den 1980er Jahren als Oberst der NVA an den streng geheimen operativen Planungen der DDR-Armee maßgeblich beteiligt. Seine Kenntnisse erweiterten das Wissen über die militärischen Planungen im Warschauer Pakt beträchtlich. So wurde deutlich, dass die sowjetische Militärführung offenbar früher als bisher angenommen, d.h. bereits vor der offiziellen Verkündung der neuen Militärdoktrin durch Michail Gorbatschow 1987, Überlegungen angestellt hatte, die bislang allein gültige Konzeption eines sofortigen strategischen Angriffs Richtung Westen zu verändern. Die Planungen sahen nunmehr in der Anfangsperiode eines Krieges zunächst eine strategische Frontverteidigungsoperation auf dem eigenen Territorium vor, von der aus dann mit einer Angriffsoperation zur endgültigen Zerschlagung des Gegners auf dessen Territorium übergegangen werden sollte. Ob diese Planungen jedoch letztlich die originäre "scharfe« Einsatzplanung des sowjetischen Generalstabes für die Vereinten Streitkräfte und auch für die NVA auf dem Westlichen Kriegsschauplatz widerspiegeln, lässt sich noch immer nicht abschließend beurteilen. Die einschlägigen Unterlagen befinden sich bis heute in Russland und sind für die Öffentlichkeit und Forschung gesperrt.

Eine Möglichkeit, die fehlende Auswertung der sowjetischen Akten wenigstens teilweise $\mathrm{zu}$ kompensieren, boten vergleichende und ergänzende Untersuchungen auf der Grundlage von Kooperationen mit renommierten Militärhistorikern aus den ehemaligen Warschauer-Pakt-Staaten. Erste Arbeiten liegen

124 Inzwischen weitergeführt als Parallel History Project on Cooperative security (PHP). Vgl. $<$ www.php.isn.ethz.ch/> (letzter Zugriff 18.1.2017).

125 Vgl. <www.wilsoncenter.org $>$ (letzter Zugriff 18.1.2017). 
inzwischen vor. In der »Grünen Reihe« ${ }^{126}$ erschienen 2009 und 2010 zwei Sammelbände mit Autoren aus dem In- und Ausland zum Warschauer Pakt. ${ }^{127}$ Sie gaben erstmals einen plastischen Eindruck von der nationalen Gemengelage im »roten« Bündnis. Die Ergebnisse bilateraler Workshops bildeten dann in der Folge die Grundlage für weitere Publikationen, die Gemeinsamkeiten, aber auch Unterschiede der nationalen Armeen und deren Rolle im Bündnis thematisierten. Den Anfang machte 2010 ein deutsch-polnischer Sammelband über die Streitkräfte der DDR und Polens. Der Polnischen Volksarmee als zweitstärkster konventioneller Streitmacht und der NVA als »Musterschüler« im Pakt kam für die Verteidigung des Ostblocks eine besondere Bedeutung $\mathrm{zu}$. In diesem Kontext ging es vor allem darum, die operativen Planungen in beiden Armeen auf dem Westlichen Kriegsschauplatz zu erhellen und in Beziehung zu setzen. Dabei wurde sichtbar, dass die unter Aufsicht der Sowjets ausgearbeiteten nationalen operativen Einsatzplanungen offenbar nicht miteinander abgestimmt waren. ${ }^{128}$

Die Rolle der Ungarischen und der Tschechoslowakischen Volksarmee im Pakt sowie ihre Beziehungen zur NVA standen im Mittelpunkt zweier weiterer Bände der Reihe »Potsdamer Schriften zur Militärgeschichte «. ${ }^{129}$ Erfreulicherweise gelang es, die Ergebnisse der gemeinsamen wissenschaftlichen Veranstaltungen teilweise zweisprachig zu veröffentlichen. ${ }^{130}$ Als weit weniger intensiv gestalteten sich die Beziehungen der NVA zur Bulgarischen Volksarmee und zu den rumänischen Streitkräften. Hierzu liegen noch keine Forschungen vor. Dessen ungeachtet gilt es, den Platz dieser Armeen im Bündnis und ihre bilateralen Beziehungen weiter im Blick zu behalten.

126 Gemeint ist die 2001 vom MGFA ins Leben gerufene Reihe »Militärgeschichte der DDR«, die seitdem mit einem charakteristischen grünen Einband im Christoph Links Verlag, Berlin, erscheint.

127 Vgl. Der Warschauer Pakt. Von der Gründung bis zum Zusammenbruch 1955 bis 1991. Im Auftrag des MGFA hrsg. von Torsten Diedrich, Winfried Heinemann und Christian F. Ostermann, Berlin 2009 (= Militärgeschichte der DDR, 16); Militär und Staatssicherheit im Sicherheitskonzept der Warschauer-Pakt-Staaten. Im Auftrag des MGFA und der Bundesbeauftragten für die Unterlagen des Staatssicherheitsdienstes der ehemaligen DDR hrsg. von Torsten Diedrich und Walter Süß, Berlin 2010 (= Militärgeschichte der DDR, 19).

128 Vgl. Die Streitkräfte der DDR und Polens in der Operationsplanung. Im Auftrag des MGFA hrsg. von Rüdiger Wenzke, Potsdam 2010 (= Potsdamer Schriften zur Militärgeschichte, 12).

129 Die NVA und die Ungarische Volksarmee (wie Anm. 8); Zwischen Bündnistreue und staatlichen Eigeninteressen. Die Streitkräfte der DDR und der ČSSR 1968 bis 1990. Im Auftrag des ZMSBw hrsg. von Oliver Bange, Potsdam 2016 (= Potsdamer Schriften zur Militärgeschichte, 26).

130 Vgl. Ivo Pejčoch, Prokop Tomek a Kolektiv, ČSLA a NLA v rámci Varšavské smlouvy, Praha 2014. 
Mit den »Beziehungsgeschichten« der NVA zur Polnischen, Ungarischen und Tschechoslowakischen Volksarmee liegt nunmehr quasi eine kleine Reihe vor, welche die jeweils nationale militärhistorische Forschung zu einigen speziellen Themen der Geschichte der sozialistischen Streitkräfte und des Warschauer Paktes aufschlussreich ergänzt. Sie ist mit neuen Forschungsergebnissen fortzusetzen, wobei die militärischen Beziehungen zwischen der NVA und der Sowjetarmee in den Mittelpunkt rücken sollten. In diesem Zusammenhang ist auch der Geschichte der sowjetischen Truppen in der DDR von 1945 bis 1994 stärkere wissenschaftliche Beachtung $\mathrm{zu}$ schenken, wenngleich sich die Quellensituation nicht geändert hat. ${ }^{131}$ Umso notwendiger sind neue Anstrengungen für eine Zusammenarbeit mit russischen Institutionen und Wissenschaftlern. Zumindest wurde 2015 am ZMSBw begonnen, den Abzug der sowjetischen Streitkräfte aus dem vereinigten Deutschland in einer wissenschaftlichen Qualifizierungsarbeit zu untersuchen. ${ }^{132}$

Worauf sollte sich das Forschungsinteresse darüber hinaus im Bereich der DDR-Militärgeschichte im Bündnis von 1945 bis 1990 in der nächsten Dekade richten? Die internationale Verflechtung und Verankerung der DDR und ihrer NVA im Warschauer Pakt, aber auch im Ost-West-Konflikt, bleiben der zentrale Untersuchungsgegenstand. Hier geht es in erster Linie um die militärische und militärtechnische Kooperation im Bündnis sowie um die Weiterführung von biund multilateralen Beziehungsgeschichten. Interessant wäre zudem zu erfahren, wie die NVA sowohl von ihren Waffenbrüdern im Bündnis als auch von ihren Gegnern im Westen fern jeder Propaganda tatsächlich beurteilt wurde.

Letztlich geht es künftig darum, die Militärgeschichte der DDR parallel zu und gemeinsam mit der Militärgeschichte der Bundesrepublik verstärkt in die deutsche Geschichte zwischen 1945 und 1990 einzuordnen. Nach ersten Einzelstudien ${ }^{133}$ bietet das am ZMSBw begonnene Großprojekt »Deutsche Militärgeschichte 1970 bis 1990 « dafür den richtungsbestimmenden Rahmen. ${ }^{134}$

131 Vgl. Hans-Albert Hoffmann und Siegfried Stoof, Sowjetische Truppen in Deutschland. Ihr Hauptquartier in Wünsdorf 1945-1994. Geschichte - Fakten - Hintergründe, Berlin 2008; Horst Lohmann, GSSD. Die Gruppe der Sowjetischen Streitkräfte in Deutschland 1945-1994. Ein historischer Abriss, Berlin 2010.

132 Vgl. das Dissertationsprojekt von Sascha Gunold (Potsdam), Der Abzug der sowjetisch-russischen Streitkräfte aus Deutschland (Arbeitstitel). Der Verfasser legt den Schwerpunkt seiner Arbeit auf die Untersuchung des Anteils deutscher Institutionen.

133 Vgl. Kontrollierte Feindschaft. Manöverbeobachtungen und Inspektionen 1987-1990. Hrsg. von Guntram König, Aachen 2011.

134 Vgl. dazu <www.mgfa-potsdam.de/html/zms_geschichteab45_2016.php> (letzter Zugriff 18.1.2017). 


\section{Militärgeschichte als Teil einer integrierten deutschen Nachkriegsgeschichte}

Im Unterschied zur Alltags- oder Konsumgeschichte, zur Sport- oder Mediengeschichte, die grenzübergreifende Untersuchungsgegenstände liefern, ${ }^{135}$ scheint sich die Militärgeschichte auf den ersten Blick gegen eine gesamtdeutsche Perspektive $\mathrm{zu}$ sperren. Gleichwohl ist $\mathrm{zu}$ klären, ob und wo sich die militärgeschichtliche Dimension im Sinne einer integrierten deutsch-deutschen Nachkriegsgeschichte zwischen »Spaltung und Verflechtung « einfügt. ${ }^{136}$

Die bisherige militärgeschichtliche Forschung hat diese grenzübergreifenden Fragen bislang auch nicht aufgeworfen, geschweige denn beantwortet. Ein kurzer Blick auf die Reihen des MGFA/ZMSBw bestätigt den Befund: Auf der einen Seite stehen die Studien zur Bundeswehrgeschichte und zur Geschichte des westlichen Bündnisses. Auf der anderen die mittlerweile 25-bändige »Militärgeschichte der DDR«, die dort, wo sie über die DDR hinausgeht, das Militärbündnis in den Blick nimmt, bestenfalls noch die befreundeten Staaten der »Dritten Welt« - nicht jedoch den anderen deutschen Staat und seine Streitkräfte. Eine Ausnahme in dieser Hinsicht ist der Band von Matthias Uhl und Armin Wagner über die westdeutsche Militärspionage in der DDR. ${ }^{137}$ Hier geht der Blick anhand der Akten des Bundesnachrichtendienstes und der Spionageabwehr des Ministeriums für Staatssicherheit über die Landesgrenze hinaus. Das Ausspähen der sowjetischen Truppen auf dem Territorium der SBZ/DDR seit den späten 1940er Jahren lässt sich als ein Modus der Wahrnehmung interpretieren. Auch wenn es um militärische

135 Vgl. Geteilte Geschichte. Ost- und Westdeutschland 1970-2000. Hrsg. von Frank Bösch, Göttingen 2015. Dazu die zurückhaltende Rezension von Christian Rau: Frank Bösch, Geteilte Geschichte. Plädoyer für eine deutsch-deutsche Perspektive auf die jüngere Zeitgeschichte. In: Zeithistorische Forschungen/Studies in Contemporary History, Online-Ausgabe, 12 (2015), H. 1, S. 98-114; vgl. auch Das doppelte Deutschland. 40 Jahre Systemkonkurrenz. Hrsg. von Udo Wengst und Hermann Wentker, Berlin 2008; Asymmetrisch verflochten? Neue Forschungen zur gesamtdeutschen Nachkriegsgeschichte. Hrsg. von Detlev Brunner, Udo Grashoff und Andreas Kötzing, Berlin 2013.

136 Vgl. Christoph Kleßmann, Spaltung und Verflechtung - Ein Konzept zur integrierten Nachkriegsgeschichte 1945 bis 1990. In: Teilung und Integration. Die doppelte deutsche Nachkriegsgeschichte. Hrsg. von Christoph Kleßmann und Peter Lautzas, Bonn 2005, S. 20-37, hier: S. 22. Noch ohne den vielzitierten Begriff: Christoph Kleßmann, Verflechtung und Abgrenzung. Aspekte der geteilten und zusammengehörigen deutschen Nachkriegsgeschichte. In: Aus Politik und Zeitgeschichte, 43 (1993), 29/30, S. 30-41, vgl. auch <www.bpb.de/apuz/29301/zwischen-abgrenzung-und-verflechtung-deutsch-deutsche-geschichte-nach-1945? $\mathrm{p}=0>$ (letzter Zugriff 18.1.2017). $137 \mathrm{Vgl}$. Uhl und Wagner, BND contra Sowjetarmee (wie Anm. 54). 
Interessen ging: $\mathrm{Zu}$ den Akteuren zählten keineswegs nur professionelle Agenten, sondern beispielsweise auch Geschäftsleute, LKW-Fahrer und Studenten, die während ihres Aufenthaltes in Ostdeutschland die Augen für den Geheimdienst in Westdeutschland offen hielten, nach Pullach berichteten - und langjährige Haftstrafen riskierten.

Selbst dort, wo es um die Schnittstelle zwischen Ost und West, die Staatsgrenze, geht, lenkt bisher eine Binnenperspektive den Blick. Neuere Studien zur Grenzpolizei und zu den Grenztruppen der $\mathrm{DDR}^{138}$ thematisieren zwar die Rolle und die Motive der »Grenzer« und zeigen sie als Instrumente der Staatsmacht, eine Studie zur »Außensicht« fehlt indes: Wie wurden die Grenztruppen, wie wurde das »Grenzregime« an der innerdeutschen Grenze und an der Berliner Mauer von außen wahrgenommen? Welche Bedeutung hatte der Grenzübertritt in der Erinnerung der Westdeutschen?

Freilich greifen manche Arbeiten der in den letzten Jahren runderneuerten, um neue Themen erweiterten »Cold War Studies « beide deutsche Staaten auf. ${ }^{139}$ Die geschichtswissenschaftliche Auseinandersetzung mit dem »Kalten Krieg « hat sich von ihrem Untersuchungsgegenstand weitgehend emanzipiert und von der Logik bipolarer Wahrnehmungen zunehmend frei gemacht. Auch wenn die Supermächte für die Forschung weiterhin eine wichtige Rolle spielen, richtet sich der Blick auch auf die außenpolitisch schwächeren Staaten und ihre Vertreter auf der politischen Bühne; davon war bereits die Rede. Vor allem aber begreifen die Cold War Studies ihren Gegenstand im Sinne einer Gesellschaftsgeschichte des Kalten Krieges: Die sozial-, kultur- und wirtschaftsgeschichtlichen Dimensionen werden als Bedingungen und Folgen politischen Handelns immer klarer ausgeleuchtet. Bernd Greiners Beobachtung, dass hier »Diplomatie- und Militärgeschichte [...] der Forschung längst nicht mehr den Takt vor[geben]«, ist insoweit zuzustimmen, als man ein enges Verständnis von Militärgeschichte zugrunde legt. ${ }^{140}$ Das trifft umso mehr zu, als im Kalten Krieg, wie Greiner selbst betont, der Übergang vom militärischen zum zivilen Bereich deshalb fließend war, weil der Feind auch im Inneren präsent und »im Frieden der Krieg, ob in der privaten Imagination oder im öffentlichen Diskurs, allgegenwärtig blieb «. ${ }^{141}$

Die längst erprobte Auffächerung der Teildisziplin, die das Blickfeld um eben jene Dimensionen erweitert hat, macht freilich die Geschichte des Kalten Krieges nach dem Kalten Krieg auch für die erweiterte Militärgeschichte interessant und

138 Vgl. Sälter, Grenzpolizisten (wie Anm. 117); Maurer, Halt - Staatsgrenze! (wie Anm. 117).

139 Siehe hierzu u.a. Grenzen überwinden (wie Anm. 21).

140 Bernd Greiner, Kalter Krieg und »Cold War Studies«, Version: 1.0. In: Docupedia-Zeitgeschichte, 11.2.2010, <http://docupedia.de/zg/Cold_War_Studies> (letzter Zugriff 18.1.2017).

141 Ebd. 
kompatibel. So wendet man sich auch hier multilateralen Konflikten einschließlich der »Dritten Welt« zu - ein Ansatz, der für eine ost- und westdeutsche Militärgeschichte beispielsweise auf einen Vergleich der militärischen Entwicklungshilfe hinauslaufen könnte. ${ }^{142}$ Das verstärkte Interesse der Cold War Studies an den kleineren, kurzfristigen Konflikten wie dem Koreakrieg, der Suez-, Kuba- und ČSSR-Krise und dem Jom-Kippur-Krieg führt auch zu der Frage, wie die politische und militärische Führung in der DDR und der Bundesrepublik mit diesen Krisen umgingen und wie sich ihr politisches Handeln gegebenenfalls gewandelt hat. Von Interesse sind nicht zuletzt jene Konfrontationen, die wie die Berlin-Krisen beide deutsche Staaten unmittelbar betroffen haben. ${ }^{143}$ In Analogie zur Weltkriegsforschung wäre auch für die Zeit nach 1945 zum einen zu fragen, wie sich die Konfliktkonstellationen auf gesellschaftliche Prozesse konkret ausgewirkt haben, zum anderen umgekehrt danach, inwieweit sozial-kulturelle Entwicklungen das politische und militärische Handeln geprägt haben. Aus dieser gespiegelten Perspektive ergeben sich wiederum Leitfragen für eine vergleichs- und verflechtungsgeschichtliche Militärgeschichte beider deutscher Staaten. Wie verschränken sich staatliche Mobilisierung und die Mobilisierung "von unten" im aufgeheizten Klima des Kalten Krieges? Auf diese Weise könnte auch eine Geschichte der Gefühle für die deutsche Militärgeschichte als Zeitgeschichte eine größere Rolle spielen, als das bislang der Fall ist. Dabei ist weiterhin eine Gesellschaftsgeschichte des Militärs, für die mittlerweile zahlreiche Studien vorliegen, von der umfassenderen Geschichte von Militär und Gesellschaft zu unterscheiden, die für Ost- und Westdeutschland, wie oben bereits ausgeführt, weiterer Forschung bedarf. Dazu gehören der Zusammenhang von politischer Legitimation und militärischer Gewalt ebenso wie die »Militarisierung « der Gesellschaft oder die »Zivilisierung des Militärs«. Dass die Antworten für die beiden unterschiedlichen Staats- und Gesellschaftsordnungen verschieden ausfallen, steht einer gemeinsamen Leitfrage nicht im Wege.

Die transnational orientierte Geschichtsschreibung hält für eine integrierte deutsche Militärgeschichte verschiedene Methoden parat: Da ist zum einen die ältere komparatistische Vorgehensweise, die ihren Gegenstand durch ein »tertium comparationis« definieren und einen systematischen Katalog von Vergleichskrite-

142 Vgl. Storkmann, Geheime Solidarität (wie Anm. 108); Klaus Storkmann, East German Military Aid to the Sandinista Government of Nicaragua, 1979-1990. In: Journal of Cold War Studies, 16 (2014), 2 (Spring), S. 56-76.

143 Vgl. Michael Lemke, Die Berlinkrisen von 1948/49 und 1958 bis 1963. In: Krisen im Kalten Krieg. Hrsg. von Bernd Greiner, Christian Th. Müller und Dierk Walter, Hamburg 2008, S. 204-244. 
rien erstellen muss. ${ }^{144}$ In einer Typologie der Vergleiche, die mit Hartmut Kaelble nach der Intention des Historikers unterscheidet, ginge es für den deutsch-deutschen Fall in erster Linie um einen »analytischen Vergleich, der Erklärungen für ein historisches Phänomen durch die gegenüberstellende Analyse unterschiedlicher Fälle entwickelt «. ${ }^{145}$

Der historische Vergleich als Methode wurde im Hinblick auf andere Methoden, die über den Nationalstaat hinausgreifen, weiterentwickelt und geöffnet. Denn die Historiker interessieren sich, wie Kaelble notiert, auch für den wechselseitigen Einfluss der verglichenen Fälle, ihre Verflechtung und die zeitgenössische Wahrnehmung der herausgearbeiteten Ähnlichkeiten oder Unterschiede zwischen den Vergleichsfällen. Zum anderen geht es deshalb um die »Verflechtungsgeschichte« (histoire croisée, entangled history ${ }^{146}$ ), die auf allerlei Ausdrucksformen einer Wechselbeziehung zielt: den Austausch von Personal oder Ideen, den Fluss von Waren oder die gegenseitige Perzeption beispielsweise.

Deshalb kann die Frage extrapoliert werden: Welche sicherheitspolitischen Antworten haben beide deutsche Staaten auf übergreifende Herausforderungen gegeben? Wirkte sich die in beiden Staaten prägende Orientierung am Westen aus, etwa in der Rüstungstechnik? Für das Militär ginge es um militärisches Know How,

144 Vgl. Vergleichen, verflechten, verwirren? Europäische Geschichtsschreibung zwischen Theorie und Praxis. Hrsg. von Agnes Arndt, Joachim C. Häberlen und Christiane Reinecke, Göttingen 2011; Comparative and transnational history. Central European approaches and new perspectives. Ed. by Heinz-Gerhard Haupt and Jürgen Kocka, New York, Oxford 2009; Hartmut Kaelble, Der historische Vergleich. Eine Einführung zum 19. und 20. Jahrhundert, Frankfurt a.M. 2009; Matthias Middell, Kulturtransfer und Historische Komparatistik - Thesen zu ihrem Verhältnis. In: Comparativ, 10 (2000), S. 7-41; Transnationale Geschichte. Themen, Tendenzen und Theorien. Hrsg. von Gunilla Budde, Sebastian Conrad und Oliver Janz, Frankfurt a.M. 2010.

145 Hartmut Kaelble, Historischer Vergleich, Version: 1.0. In: Docupedia-Zeitgeschichte, 14.8.2012, <https://docupedia.de/zg/Historischer_Vergleich> (letzter Zugriff 23.1.2017). Kaelble nennt ferner »den kontrastiven aufklärenden Vergleich, der sich etwa mit der Entwicklung der Demokratie oder den Menschenrechten befassen kann und ihrer historischen Durchsetzung in den einen Ländern, ihr historisches Scheitern in anderen Ländern gegenüberstellt und erklärt; den verstehenden und gleichzeitig distanzierenden Vergleich, der andere Länder durch den historischen Vergleich mit dem eigenen Land besser zu verstehen versucht und gleichzeitig damit auch das historische Selbstverständnis des eigenen Landes aus anderer Perspektive sieht und revidiert.«Vgl. auch Kaelble, Der historische Vergleich (wie Anm. 144); vgl. für einen diachronen und synchronen Vergleich: Jörg Echternkamp, Der politische Offizier als normativer Typus. Zum Verhältnis von Politik und Militär im »Dritten Reich«, in der DDR und der Bundesrepublik. In: Deutsche Offiziere. Militarismus und die Akteure der Gewalt. Hrsg. Von Galili Shahar, Göttingen 2016 (= Tel Aviver Jahrbuch für Geschichte, 44), S. 221-250.

146 Vgl. Michael Werner und Bénédicte Zimmermann, Vergleich, Transfer, Verflechtung. Der Ansatz der Histoire croisée und die Herausforderung des Transnationalen. In: Geschichte und Gesellschaft, 28 (2002), 4, S. 607-636. 
die Konstruktion von Selbst- und Feindbildern in NVA und Bundeswehr oder sofern auf die DDR übertragbar - um zeitgenössische Selbstbeschreibungen, etwa in der Militärsoziologie oder Militärpresse, am Ende auch um Manöverbeobachtungen. Beide Ansätze schließen sich daher nicht länger aus, sondern können einander ergänzen, nicht zuletzt in einer komparativen Perzeptionsgeschichte. Wo im konkreten Fall der Schwerpunkt liegt, ob der analytische Vergleich im Vordergrund steht oder die Verflechtung, hängt vom Einzelfall, vom Erkenntnisinteresse des Historikers wie von der Quellenlage ab.

Auch eine gesamtdeutsche Militärgeschichte der 1970er und 1980er Jahre wird um das spezifische Spannungsverhältnis nicht herumkommen, das in der Ungleichartigkeit und im Ungleichgewicht von DDR und Bundesrepublik angelegt war. Christoph Kleßmann hat das mit dem vielzitierten Paradoxon einer »asymmetrisch verflochtene Parallelgeschichte ${ }^{147}$ auf den Begriff gebracht. Inwieweit bildete die Bundeswehr einen Bezugsrahmen für die NVA? Hat die NVA etwa die Bundeswehr beeinflusst? So lautet die Leitfrage einer gesamtdeutschen Geschichte, wenn man sie auf die Militärgeschichte herunterbricht. Gab es nicht auch in der westdeutschen Militär- und Sicherheitspolitik, in der militärischen Praxis oder im Kasernenalltag zwischen Braunschweig und Bonn Bereiche, die ohne die Existenz der anderen deutschen Streitkräfte anders ausgesehen hätten? Diese Fragen zeigen, dass die integrale Betrachtung eine unterschiedliche, asymmetrische Gewichtung nicht ausschließt.

Auch für eine gesamtdeutsche Militärgeschichte als Vergleichs- und Verflechtungsgeschichte ist ein umfassendes Verständnis der Teildisziplin Militärgeschichte unabdingbar, wie es sich seit den 1990er Jahren nicht nur in der Erforschung der Militärgeschichte von Ost- und Westdeutschland durchgesetzt hat und als Leitlinie künftiger Arbeiten gefordert wurde. ${ }^{148}$

Der Blick auf das Ende der DDR darf dabei nicht die Sicht auf die Phasen ihrer relativen Stabilität verstellen. Ebenso wenig sollte die Stabilität der westdeutschen Streitkräfte, die sich nicht zuletzt zur Verblüffung ihrer Kritiker als eine Ordnungsmacht der westdeutschen Demokratie erwiesen, über innere Verwerfungen hinwegtäuschen, wie sie sich intern im Dauerstreit über die Führungsphilosophie der Inneren Führung zeigten und nach außen in der Kritik am Militär - vor allem während der Nachrüstungsdebatte in den frühen 1980er Jahren - artikulierte. Mit dieser Maßgabe wäre $\mathrm{zu}$ fragen: Welche Rolle spielten

147 Vgl. dazu u.a. Christoph Kleßmann, Hans Misselwitz und Günter Wichert, Vorwort. In: Deutsche Vergangenheiten - eine gemeinsame Herausforderung. Der schwierige Umgang mit der doppelten Nachkriegsgeschichte. Hrsg. von Christoph Kleßmann, Hans Misselwitz und Günter Wichert, Berlin 1999, S. $12 \mathrm{f}$.

148 Vgl. Bröckermann [u.a.], Die Zukunft der DDR-Militärgeschichte (wie Anm. 7), S. 72. 
die Streitkräfte für die Legitimierung und Delegitimierung der ost- bzw. westdeutschen Staats- und Gesellschaftsordnung? Welche Bedeutung besaßen in diesem Zusammenhang die jeweiligen Militärallianzen, die Bündnispartner und die Supermächte UdSSR bzw. USA?

Die methodischen Fallstricke liegen parat. Da Militärhistoriker heute gleichsam von einem westdeutschen Standpunkt aus forschen, lauern mindestens drei Gefahren: dass sie ihren Untersuchungsgegenstand an Kriterien der »eigenen« Militärpolitik oder militärischen Alltagskultur festmachen und auf die DDR projizieren; dass sie die ostdeutsche Militärgeschichte als Verfallsgeschichte konzipieren und teleologisch auf die Wiedervereinigung zulaufen lassen; dass Wechselbeziehungen zwischen NVA und Bundeswehr vor lauter Begeisterung über den Nachweis des Transnationalen zu stark gewichtet werden.

Eine gesamtdeutsche Militärgeschichte $\mathrm{zu}$ schreiben ist sinnvoll, weil der gemeinsame Erfahrungshintergrund die (nur) vierzigjährige Geschichte geprägt hat und beide deutsche Gesellschaften aufgrund der gemeinsamen Sprache, der familiären Kontakte und des grenzübergreifenden Medienkonsums als eine »Kommunikationsgemeinschaft « ${ }^{149}$ verstanden werden können. Im günstigen Fall bietet auch die deutsch-deutsche Militärgeschichte als jüngere Zeitgeschichte im internationalen Kontext drei Vorzüge: Inhaltlich bereichert sie eine gesamtdeutsche Nachkriegsgeschichte um Erkenntnisse auf einem zentralen Gebiet staatlicher Souveränität: dem Militär. Theoretisch befeuert sie die Methodendiskussion: in der Zeitgeschichte wie in der Militärgeschichte selbst. Sie weist neue Wege, die über die vertrauten Pfade der Militärgeschichte der Bundesrepublik und der DDR hinausführen. Politisch hilft der übergreifende Blick auf die Jahrzehnte vor 1990 besser $\mathrm{zu}$ verstehen, welche Schwierigkeiten das Zusammenwachsen bereitete und welche Unterschiede bis heute bestehen. Darin liegt eine Chance von Militärgeschichte als Problemgeschichte der Gegenwart.

Die vergleichende Betrachtung könnte eine noch genauere Antwort auf die Frage geben, warum wir es in der DDR mit einer weitgehend militarisierten Gesellschaft zu tun haben, während in der Bundesrepublik bei gleichen historischen Voraussetzungen die Haltung gegenüber dem Militär zwischen Affinität und Ablehnung schwankte. Weder die NVA noch die Bundeswehr können aus dem gesamtgesellschaftlichen Entwicklungsprozess herausgelöst werden, der sie prägte und den sie prägten. Zugleich könnte der Vergleich ihres Handelns im Bündnis nicht nur deren strukturelle Bedingungen zeigen, sondern auch verdeut-

149 Vgl. dazu u.a. Axel Schildt, Die Sozialgeschichte der Bundesrepublik Deutschland bis 1989/ 90, München 2007 (= Enzyklopädie Deutscher Geschichte, 80), S. 85. 
lichen, welche Rolle die Deutschen im Ost-West-Konflikt gespielt haben. Dieser Vorschlag ist nicht neu, aber weiterhin aktuell.

\section{Deutsche Militärgeschichte 1970 bis 1990 - ein aktuelles Forschungsprojekt}

Mit einem großen, 2014 begonnenen interdisziplinären Forschungsprojekt hat das ZMSBw den Versuch unternommen, die Nachkriegsgeschichte der beiden deutschen Staaten am Beispiel der Militärgeschichte zusammenzuführen. ${ }^{150}$ Erstmals soll eine gemeinsame, in die Entwicklung der Militärbündnisse eingebundene deutsche Militärgeschichte geschrieben werden. Die Teilprojekte beleuchten vier historische Problemfelder: die militärischen Binnenstrukturen, das militärischzivile Verhältnis, den sicherheits- und militärpolitischen Wandel sowie die rüstungswirtschaftlichen Entwicklungen. Methodisch greifen sie dazu in erster Linie auf die genannten Analysekategorien Vergleich und Transfer zurück, wie sie vor allem im deutsch-französischen Wissenschaftskontext entwickelt und für die Geschichte der Kulturbeziehungen fruchtbar gemacht worden sind. ${ }^{151}$

Im Hinblick auf die Binnenstrukturen von Bundeswehr und NVA bieten sich, erstens, folgende Aspekte für einen Vergleich an: die Organisationsstrukturen, Bewaffnung und Ausrüstung sowie Prinzipien der operativen Planung; Konzepte und Grundsätze der Menschenführung, der Erziehung und Ausbildung, der Disziplinarverhältnisse und der Traditionspflege, aber auch der Alltag der Soldaten in den Kasernen und außerhalb des Dienstes. Als ein weiterer Indikator für die Menschenführung und das Menschenbild soll zudem die sanitätsdienstlich-medi-

150 Projektleitung: Rüdiger Wenzke, Koordination: Jörg Echternkamp. Zu Details vgl. <www. mgfa-potsdam.de/html/zms_geschichteab45_2016.php> (letzter Zugriff 18.1.2017). Aus Anlass des 25. Jahrestags der deutschen Einheit entstand 2015 die Ausstellung »Militär und Gesellschaft in Deutschland seit 1945«. Diese Ausstellung des ZMSBw und der Bundesstiftung zur Aufarbeitung der SED-Diktatur befasst sich mit dem Platz der Streitkräfte sowohl in der Bundesrepublik als auch in der DDR. Vgl. <www.bundesstiftung-aufarbeitung.de/militaer-und-gesellschaft-indeutschland-seit-1945-5047.html $>$ (letzter Zugriff 18.1.2017).

151 Zwischen Transfer und Vergleich. Theorien und Methoden der Literatur- und Kulturbeziehungen aus deutsch-französischer Perspektive. Hrsg. von Christiane Solte-Gresser, Hans-Jürgen Lüsebrink und Manfred Schmeling, Stuttgart 2013 (= Vice Versa, 5). Zur Verbindung der nationalgeschichtlichen Ebene mit transfergeschichtlichen und transnationalen Ansätzen, v. a. für die Zeit seit den 1970er Jahren, vgl. Deutschland und Frankreich in der Globalisierung im 19. und 20. Jahrhundert. Hrsg. von Jean-François Eck und Dietmar Hüser, Stuttgart 2012 (= Schriftenreihe des Deutsch-Französischen Historikerkomitees, 8). 
zinische Versorgung im Rahmen der Entwicklung des Sanitätsdienstes der Bundeswehr und des Medizinischen Dienstes der Nationalen Volksarmee verglichen werden. Darüber hinaus findet im ersten Untersuchungsschwerpunkt einer künftigen »Deutschen Militärgeschichte von 1970 bis 1990« die militärische Elite durch einen Vergleich der Heeresgenerale beider Armeen im Hinblick auf ihre soziale Herkunft und ihre Karrierewege besondere Aufmerksamkeit.

Zweitens geht es um Dimensionen des zivil-militärischen Verhältnisses in der DDR und der Bundesrepublik. Der Blick richtet sich zum einen politikgeschichtlich auf die Politisierung des Militärs und die Streitkräfte als politische Handlungsfelder. Die Annäherung der Gewerkschaften an die Bundeswehr wird hier eine Rolle spielen. Zum anderen interessiert wissenschaftsgeschichtlich die Organisation der militärgeschichtlichen Forschung in der Bundeswehr und der NVA, die deutsch-deutschen wie internationalen Kontakte sowie die Selbstbeschreibungen durch die noch junge Militärsoziologie. $\mathrm{Zu}$ den zivil-militärischen Problemfeldern der 1970er und 1980er Jahre gehört nicht zuletzt die im Kontext der Nachrüstungsdebatte heftig umstrittene Frage, wie der Friede gewahrt werden und welche Rolle das Militär dabei spielen könne. Die beiden christlichen Kirchen und die »Friedensbewegungen« in Ost- und Westdeutschland kommen damit in den Fokus. Auch hier geht es um Vergleich, Austausch und internationale Zusammenhänge. Nicht nur im vorpolitischen Bereich, auch in der politischen Arena im engeren Sinn drehte sich viel um militär- und sicherheitspolitische Fragen. Deshalb eignen sich, so lautet eine weitere Annahme, auch die verschiedenen verteidigungspolitischen Akteure im parlamentarischen Raum - von einzelnen Politikern über die Parteien und Ausschüsse - für eine vergleichende Betrachtung. Wie weit führten die entgegengesetzten politischen Systembedingungen zu unterschiedlichen oder analogen Formen der Organisation und Vermittlung verteidigungspolitischer Ziele über das Parlament hinaus? In beiden deutschen Staaten hing die Verteidigungsbereitschaft nicht zuletzt von der Einstellung der Bürgerinnen und Bürger gegenüber der Sicherheitspolitik der jeweiligen Regierung und den eigenen Streitkräften ab. Daher ist der (sozialwissenschaftlich orientierte) Vergleich des sicherheitspolitischen Meinungsbildes Teil der historischen Untersuchung des zivil-militärischen Problemfeldes.

Für eine Vergleichsgeschichte, weniger eine Verflechtungsgeschichte, eignet sich, drittens, der Zusammenhang von Militär und Rüstungswirtschaft. Im Schnittpunkt von Militär- und Wirtschaftsgeschichte soll daher der Frage nachgegangen werden, wie beide deutsche Staaten ihre Streitkräfte militärisch ausgerüstet haben. Wie regelten Militär, Wirtschaft und Politik unter den unterschiedlichen politischen und ökonomischen Bedingungen die Rüstungsgüterbeschaffung? Auch hier kommt es auf die nationalen Möglichkeiten unter den Bedingungen der internationalen Bündniskonstellation an. Der Blick »von außen« auf diese bünd- 
nis- und sicherheitspolitischen Rahmenbedingungen lässt schließlich, viertens, ein Handlungsfeld erkennen, das die Notwendigkeit einer internationalen Kontextualisierung der deutsch-deutschen Geschichte in den letzten zwanzig Jahren des Ost-West-Konflikts herausstellt. Die Militärpolitik der Bundesrepublik wie der DDR lassen sich ohne die Entwicklung der internationalen Beziehungen kaum verstehen. Es bleibt abzuwarten, inwieweit diese außenpolitischen Entwicklungen zu den innenpolitischen Veränderungen in ein Verhältnis gesetzt werden können.

Die Teilprojekte können als Bausteine einer integralen deutschen Militärgeschichte in den letzten zwanzig Jahren des Ost-West-Konfliktes verstanden werden, die im Idealfall nicht zuletzt durch ihre sozial-, kultur-, politik- und wirtschaftsgeschichtliche Öffnung gegenüber den zivil-militärischen Beziehungen Anknüpfungspunkte zu anderen laufenden Kontroversen in der neueren Zeitgeschichte bietet. Indem die Studien auf vergleichs- und verflechtungsgeschichtliche Ansätze zurückgreifen, testet das Projekt ihren Ertrag für die neuere Zeitgeschichte anhand des empirischen Beispiels der deutsch-deutschen Militärgeschichte. Der Ansatz, die jüngere Zeitgeschichte "nach dem Boom« als Vorgeschichte der Gegenwart zu betrachten, ${ }^{152}$ verspricht zum einen Antworten auf die Frage nach den Bedingungen der Wiedervereinigung. Zum anderen werden historisch bedingte Gemeinsamkeiten und Unterschiede als Aspekte des Einigungsprozesses deutlich. Dass diese Themen auf großes Interesse stoßen, hat ein öffentlicher Workshop ${ }^{153}$ im März 2016 in Potsdam gezeigt.

Vor zehn Jahren, als an dieser Stelle schon einmal über die Zukunft der Militärgeschichtsschreibung, wenn auch nur auf die DDR beschränkt, diskutiert wurde, lautete die Alternative: »Einbettung der DDR-Geschichte in übergreifende Perspektiven« oder »die DDR selbst ernst nehmen? «. ${ }^{154}$ Einig war man sich darin, dass die Forschung zur deutschen Militärgeschichte nach 1945 neue Impulse benötigte. Der »deutsch-deutsche« Ansatz ist solch ein Impuls, weil er einen gangbaren Mittelweg zwischen der weiten, transnationalen und der engeren deutschen Forschungslandschaft eröffnet. Grundlagenforschung zur ost- und westdeutschen Militärgeschichte und $\mathrm{zu}$ den Bündnissen bleibt dafür eine Voraussetzung.

152 Vgl. Anselm Doering-Manteuffel und Lutz Raphael, Nach dem Boom. Perspektiven auf die Zeitgeschichte seit 1970, 3., erg. Aufl. Göttingen 2012.

$153 \mathrm{Vgl}$.<www.hsozkult.de/conferencereport/id/tagungsberichte-6520> (letzter Zugriff 18.1.2017) Leonie Ziegler, Tagungsbericht zum Workshop »Gibt es eine deutsch-deutsche Militärgeschichte als neuere Zeitgeschichte?«, Potsdam, 9.3.2016, vgl. auch den Tagungsbericht in diesem Heft, S. $181-189$.

154 Bröckermann [u.a.], Die Zukunft der DDR-Militärgeschichte (wie Anm. 7), S. 74. 